Article

\title{
Ammonium to Total Nitrogen Ratio Interactive Effects with Salinity Application on Solanum lycopersicum Growth, Physiology, and Fruit Storage in a Closed Hydroponic System
}

\author{
Nikolaos Tzortzakis ${ }^{1,2, *(D)}$, Georgia Pitsikoulaki ${ }^{2}$, Aristeidis Stamatakis ${ }^{3}$ and Antonios Chrysargyris ${ }^{1}$ \\ 1 Department of Agricultural Sciences, Biotechnology and Food Science, Cyprus University of Technology, \\ Lemesos 3603, Cyprus; a.chrysargyris@cut.ac.cy \\ 2 Department of Sustainable Agriculture, Mediterranean Agronomic Institute of Chania, 73100 Chania, Greece; \\ georgia.pitsikoulaki@gmail.com \\ 3 Max Planck Institute for Plant Breeding Research, D-50829 Koln, Germany; stamatakis@mpipz.mpg.de \\ * Correspondence: nikolaos.tzortzakis@cut.ac.cy; Tel.: +357-25002280
}

\section{check for}

updates

Citation: Tzortzakis, N.

Pitsikoulaki, G.; Stamatakis, A.;

Chrysargyris, A. Ammonium to Total Nitrogen Ratio Interactive Effects with Salinity Application on Solanum lycopersicum Growth, Physiology, and Fruit Storage in a Closed Hydroponic System. Agronomy 2022, 12, 386. https:// doi.org/10.3390/agronomy12020386

Academic Editor: Alberto San Bautista

Received: 11 January 2022

Accepted: 1 February 2022

Published: 3 February 2022

Publisher's Note: MDPI stays neutral with regard to jurisdictional claims in published maps and institutional affiliations.

Copyright: (C) 2022 by the authors. Licensee MDPI, Basel, Switzerland. This article is an open access article distributed under the terms and conditions of the Creative Commons Attribution (CC BY) license (https:// creativecommons.org/licenses/by/ $4.0 /)$

\begin{abstract}
Using high-salinity water for plant fertigation may have negative consequences for plant growth, overall yield and crop quality. In the present study, the effects of $\mathrm{NaCl}$-salinity in conjunction with three different ammonium to nitrate ratios (Nr) on tomato (Solanum lycopersicum Mill.) plant growth, nutritional status, yield, fruit quality and postharvest storage were examined. The electrical conductivity (EC) was increased by adding $\mathrm{NaCl}$ into the nutrient solution and three different $\mathrm{Nr}$ ratios were applied, $\mathrm{Nr} 0.05, \mathrm{Nr} 0.10$ and $\mathrm{Nr} 0.15$, while the other macronutrient and micronutrient concentrations were constant in all treatments. The EC of the nutrient solution supplied to the plants was $2.2 \mathrm{mS} \mathrm{cm}^{-1}$ at the low salinity treatments and $7.5 \mathrm{mS} \mathrm{cm}^{-1}$ at the high salinity treatments. Increased salinity resulted in decreased plant growth factors and fruit yield, despite the Nr. An increased $\mathrm{Nr}$ reduced the $\mathrm{pH}$ value of the nutrient solution, while the fruits of the plants cultivated under high salinity obtained reduced $\mathrm{Ca}, \mathrm{K}, \mathrm{Mg}, \mathrm{P}$ and $\mathrm{N}$ content. Reduced Ca content can lead to blossom end rot disorder and this was evidenced on tomato cultivation under high salinity. However, in the present study, this disorder appeared at the very late stages and did not affect the marketability of the fruits. On the other hand, both salinity and Nr0.15 increased fruit firmness at harvest or following storage, while citric acid, total soluble solid and vitamin $C$ contents were also increased by salinity. Total phenols were increased by salinity at harvest, and lycopene and $\beta$-carotene content were increased by salinity at harvest and/or following storage at $12{ }^{\circ} \mathrm{C}$ or $25^{\circ} \mathrm{C}$. Salinity enhances fruit quality and improves the organoleptic characteristics of the crop, while an appropriate $\mathrm{Nr}$ ratio may restrict the detrimental effects of salinity on the nutritional status of plants by regulating the $\mathrm{pH}$ in hydroponic systems.
\end{abstract}

Keywords: minerals; fruit quality; salinity stress; nitrogen form; soilless culture; storage; tomato

\section{Introduction}

Salinity and a lack of high-quality irrigation water are two key side effects of ongoing climate change that have a negative impact on plant physiological processes, plant growth and agronomic performance, with serious implications for global horticulture crop output $[1,2]$. According to reports, an increase in water and soil salinity is causing problems in $20 \%$ of agricultural and $33 \%$ of irrigated lands around the world $[3,4]$. Increased salt levels in soil solution are known to raise osmotic pressure in the rhizosphere, resulting in a reduction in water availability and nutrient uptake by plants [5]. Salinity stress has also been shown to have a deleterious impact on plant physiological and biochemical processes, affecting both the primary and secondary metabolism of plants [6]. Increasing sodium chloride $(\mathrm{NaCl})$ levels has been demonstrated to have a negative effect on plant height and vigor [7-9], leaf area [10,11] and leaf number $[12,13]$. High salinity also reduces 
plant photosynthetic rate [14], which can alter protein content [15], which in turn alters quality and activates many plant secondary metabolic processes [16], as well as antioxidant mechanisms to combat salinity impacts $[17,18]$.

Nitrogen $(\mathrm{N})$ is the sole nutrient that can be delivered to plants in both anionic (nitrate; $\mathrm{NO}_{3}{ }^{-}$) and cationic (ammonium; $\mathrm{NH}_{4}^{+}$) form by fertigation, and both $\mathrm{N}$ forms have different absorption rates depending on their external concentrations [19]. Plant responses to $\mathrm{NH}_{4}{ }^{+}$vary by species and are influenced by environmental factors such as temperature, light intensity, $\mathrm{pH}$ and nutrient concentrations in the growth media [20]. The form of $\mathrm{N}$ used by a plant may affect its morphology and chemical composition, with $\mathrm{NH}_{4}{ }^{+}$likely to cause lower levels of potassium $(\mathrm{K})$, calcium $(\mathrm{Ca})$ and magnesium $(\mathrm{Mg})$ and higher levels of phosphorus (P), sulphur (S) and organic N [21]. Several studies have found that the form of nitrogen, rather than the total nitrogen amount, is more important, with major implications not only for the overall yield but also for the marketable quality of the final vegetable products [22-24]. As a result, changing the composition of the nutrient solution (NS) can be a cost-effective way to improve vegetable quality by reducing antinutritional components [25] or increasing the beneficial compounds levels [26]. Most plants exhibit growth reduction and severe toxicity symptoms if $\mathrm{NH}_{4}{ }^{+}$is the dominant or sole form of the supplied N, due to the intracellular concentration of ammonia, which is highly toxic for the plant cells [19], being increased as result. Despite the fact that the energetic cost of $\mathrm{NO}_{3}{ }^{-}$absorption and assimilation is significantly higher than that of $\mathrm{NH}_{4}{ }^{+}$, the majority of plant species prefer $\mathrm{NO}_{3}{ }^{-}$to $\mathrm{NH}_{4}{ }^{+}$[27]. This is because large concentrations of $\mathrm{NH}_{4}{ }^{+}$in the solution $(>0.5 \mathrm{mM})$ can be harmful to plants (particularly at low $\mathrm{pH}$ levels), reducing biomass production $[27,28]$. The concentration of nitrate in edible tissues, on the other hand, is an essential quality attribute of vegetables. Nitrate levels in vegetables are frequently addressed as a potential health hazard. Furthermore, nitrite can react with amines and amides in the body to generate nitrosamines and nitrosamides, both of which have been linked to cancer [22].

Although various studies have demonstrated that high salinity has a negative impact on plant growth and fruit yield, tomato is regarded as a species which is fairly resilient to salt stress $[29,30]$. However, there is still a gap in our understanding of the plant physiology parameters, biochemical alterations and postharvest performance of saline-grown tomato plants in combination with nitrogen forms and ratios, for example, the ratio of ammonium ions to total nitrogen (Nr: $\mathrm{NH}_{4}{ }^{+} /$total $\mathrm{N}$ ). Several studies have involved a broad spectrum of $\mathrm{Nr}$ ranging from 0 to 1 [31] but this has not enabled an accurate optimization of the $\mathrm{Nr}$ responses. Therefore, interest has been focused on a narrower range of $\mathrm{Nr}$ [32]. As a result, this study's main objectives were to: (a) investigate the effects of the application of ammonium to total nitrogen ratios as well as salinity conditions via nutrient solution on tomato plant growth, mineral content and fruit quality; (b) assess the postharvest performance of fruits when plants were subjected to $\mathrm{Nr}$ or saline conditions under storage and shelf-life conditions. The findings of this study could lead to a better knowledge of plant responses to salinity and/or Nr application for the purpose of using hydroponic nutrient solution to aid in tomato plant development and fruit shelf-life.

\section{Materials and Methods}

\subsection{Plant Material and Growing Conditions}

The present study was carried out in a run-to-waste system hydroponic Venlo doubleroof type glasshouse with a North-South orientation, located at the Mediterranean Agronomic Institute of Chania, Greece, at the latitude of $35.35^{\circ} \mathrm{N}$, longitude of $24.02^{\circ} \mathrm{E}$ and altitude of $8 \mathrm{~m}$. The study took place during the autumn-spring growing period (from the middle of October until the end of March in 2014-2015). Air temperature and relative humidity were averaged from $15.3{ }^{\circ} \mathrm{C}$ to $24.6^{\circ} \mathrm{C}$ and $62.1-89.5 \%$, respectively (Figure S1).

Tomato seedlings (Solanum lycopersicum Mill. cv. Belladonna) grown in standard potting mix were purchased from a local nursery. Seedlings were transplanted into perlite filled bags (three seedlings per bag, spaced at $33 \mathrm{~cm}$ ) at the two true-leaf stage. 


\subsection{Experimental Set Up}

Seedlings were given a seven-day period to become acclimatized to the new growing media (perlite). Six treatments were examined, considering three different $\mathrm{NH}_{4}-\mathrm{N} / \mathrm{N}_{\text {total }}$ supply ratios $(\mathrm{Nr})$ in conjunction with two salinity levels (three $\mathrm{N}$ ratio $\times$ two salinity $\times$ four replication plots). The treatments were named as plants grown with (i) Nr0.05 + LS $\left(\mathrm{NH}_{4}-\mathrm{N} / \mathrm{N}_{\text {total }}\right.$ : 0.05 , low salinity of $\left.2.2 \mathrm{mS} \mathrm{cm}^{-1}\right)$, (ii) $\mathrm{Nr} 0.10+\mathrm{LS}\left(\mathrm{NH}_{4}-\mathrm{N} / \mathrm{N}_{\text {total }}\right.$ : 0.10 , low salinity of $\left.2.2 \mathrm{mS} \mathrm{cm}^{-1}\right)$, (iii) $\mathrm{Nr} 0.15+\mathrm{LS}\left(\mathrm{NH}_{4}-\mathrm{N} / \mathrm{N}_{\text {total }}\right.$ : 0.15 , low salinity of $\left.2.2 \mathrm{mS} \mathrm{cm}^{-1}\right)$, (iv) $\mathrm{Nr} 0.05+\mathrm{HS}\left(\mathrm{NH}_{4}-\mathrm{N} / \mathrm{N}_{\text {total }}: 0.05\right.$, high salinity of $\left.7.5 \mathrm{mS} \mathrm{cm}^{-1}\right)$, (v) $\mathrm{Nr} 0.10+\mathrm{HS}\left(\mathrm{NH}_{4}-\mathrm{N} / \mathrm{N}_{\text {total }}: 0.10\right.$, high salinity of $\left.7.5 \mathrm{mS} \mathrm{cm}^{-1}\right)$ and (vi) $\mathrm{Nr} 0.15+$ HS $\left(\mathrm{NH}_{4}-\mathrm{N} / \mathrm{N}_{\text {total }}: 0.15\right.$, high salinity of $\left.7.5 \mathrm{mS} \mathrm{cm}^{-1}\right)$. For the purposes of our study, we opted to use the moderate salinity level of $50 \mathrm{mM} \mathrm{NaCl}$ in order to avoid subjecting plants to severe stress that could not allow fruit formation and the harvesting of fruit due to early plant death. Therefore, the term salinity is used to differentiate the experimental treatments $(0$ and $50 \mathrm{mM} \mathrm{NaCl})$. To achieve the targeted salinity conditions in treatments (iv), (v) and (vi), electrical conductivity (EC) was gradually increased with the addition of $\mathrm{NaCl}$ over a nine-days period (from 2.20 for standard NS up to $7.50 \mathrm{mS} \mathrm{cm}^{-1}$ for salinity treatment) to avoid osmotic shock. The different $\mathrm{Nr}$ supply was on molar basis. Six tanks containing the six different nutrient solutions used for fertigation, with each tank being $300 \mathrm{~L}$ in volume, were established in the glasshouse. The composition of macronutrients in standard NS $(1: 100 \mathrm{v} / \mathrm{v})$ was: $\mathrm{NO}_{3}{ }^{-}-\mathrm{N}=14.96 \mathrm{mmol} \mathrm{L}{ }^{-1}$, $\mathrm{K}=8.75 \mathrm{mmol} \mathrm{L}^{-1}, \mathrm{PO}_{4}^{-3}-\mathrm{P}=1.07 \mathrm{mmol} \mathrm{L}^{-1}, \mathrm{Ca}=7.22 \mathrm{mmol} \mathrm{L}^{-1}, \mathrm{Mg}=3.04 \mathrm{mmol} \mathrm{L}^{-1}$, $\mathrm{SO}_{4}{ }^{-2}-\mathrm{S}=3.37 \mathrm{mmol} \mathrm{L}^{-1}$ and sodium $(\mathrm{Na})=0.34 \mathrm{mmol} \mathrm{L}^{-1}$; while the micronutrient contents were: boron $(B)=25.00 \mu \mathrm{mol} \mathrm{L}{ }^{-1}$, iron $(\mathrm{Fe})=20.00 \mu \mathrm{mol} \mathrm{L}^{-1}$, manganese $(\mathrm{Mn})=10.00 \mu \mathrm{mol} \mathrm{L}-1$, copper $(\mathrm{Cu})=0.77 \mu \mathrm{mol} \mathrm{L}^{-1}$, zinc $(\mathrm{Zn})=4.00 \mu \mathrm{mol} \mathrm{L}^{-1}$ and molybdenum $(\mathrm{Mo})=0.50 \mu \mathrm{mol} \mathrm{L^{-1 }}$. The $\mathrm{pH}$ of NS treatment was adjusted at optimal levels (5.6) for both types of solutions with the addition of $\mathrm{HNO}_{3}(0.9 \% v / v)$ and the ratio of $\mathrm{N} / \mathrm{P} / \mathrm{K}$ was constant in all treatments. Fresh NS was prepared twice weekly, according to fertigation needs, while $\mathrm{pH}$ and $\mathrm{EC}$ levels were checked and adjusted whenever a new solution was prepared. Fertigation was applied via a drip irrigation system seven times per day (at full development stage) for three minutes per application, with a total volume of $777 \mathrm{~mL}$ of solution applied daily per plant. The fertigation schedule was adjusted from time to time depending on the plant developmental stage and the current environmental conditions. In general, an effort was made to collect $25 \%$ of the drainage solution from the nutrient solution that was applied.

The experiment was laid out as a completely randomized design, with four replication plots for each of the six treatments and six plants per replication plot, giving us a total of twenty-four plants per treatment, out of which sixteen individual plants (four plants per replication) were considered as experimental units and subjected to further measurements.

Plants were grown in bags filled with perlite as a growing media. The bags were placed on the metal channels, forming planting rows which had a distance of $0.8 \mathrm{~m}$. The central corridor was $1.3 \mathrm{~m}$ wide. Three tomato plants (Solanum lycopersicum Mill. cv. Belladonna) were planted in each bag, spaced $0.33 \mathrm{~m}$ apart. The bags had slits on the bottom to allow drainage. The first fruit set was 4 weeks and the first harvest took place after 12 weeks after transplanting.

A vertical single stem pruning system was applied to the plants and pruning was performed almost every week. Sulfur was spread on the glasshouse floor during the cultivation period in order to protect the crop from pathogen infection. Crop protection was performed with the use of approved insecticides (AFFIRM, Decis) against Tuta absoluta (considered the most serious pest enemy for tomato cultivation in Crete) every 10 days. Additional sprayings with Switch and Mepam were performed three times during the cultivation period against Botrytis cinerea with the appearance of the first symptoms (middle January). Additionally, yellow sticky traps were hung for protection from pests (Bemisia tabaci).

Pollination was performed with bumble bees that were established in the glasshouse for the whole growing period. The bumble bees were applied twice during cultivation. The 
insecticides used against T. absoluta were always applied during the late afternoon; by that time the bees had already entered the beehive. The beehive remained closed until the next day to protect the bumble bees from the insecticide. The beehive was removed from the glasshouse before the spraying of fungicides.

\subsection{Plant Growth and Physiological Parameters}

Forty-eight tomato plants (eight plants per treatment) were marked and used for plant growth measurements. Plant growth characteristics were recorded once, at the end of the study (12 weeks after transplanting). The recorded parameters included plant height $(\mathrm{cm})$, number of leaves, stem diameter $(\mathrm{cm})$, number of fruits, fresh fruit weight $(\mathrm{g})$ and total yield $(\mathrm{kg})$.

Leaf chlorophyll was extracted with dimethyl sulfoxide (DMSO) and Chlorophyll a (Chl a), Chlorophyll b ( $\mathrm{Chl} b)$ and total Chlorophylls (total $\mathrm{Chl}$ ) were assayed and expressed as $\mu \mathrm{g} \mathrm{g}^{-1} \mathrm{Fw}$ [33]. Leaf photosynthetic rate $(P \mathrm{n})$, stomatal conductance $(g \mathrm{~s})$ and internal leaf concentration of $\mathrm{CO}_{2}(\mathrm{Ci})$ were recorded with a $\mathrm{Li}$-Cor gas analyzer $(\mathrm{Li}-$ 6400, Li-Cor, Inc., Lincoln, NE, USA). All gas exchange measurements started $3 \mathrm{~h}$ after the onset of the photoperiod with the following instrumental adjustments: leaf chamber $\left(6 \mathrm{~cm}^{2}\right)$, temperature $26 \pm 2{ }^{\circ} \mathrm{C}$ and photon flux density $1300 \mu \mathrm{mol} \mathrm{m}{ }^{-2} \mathrm{~s}^{-1}$ at ambient $\mathrm{CO}_{2}$ concentration. For each treatment, measurements were taken from two fully expanded, healthy and sun-exposed leaves per plant from four plants in total.

\subsection{Fruit Harvesting}

For yield estimation, fruit were harvested from the first four clusters of selected tomato plants. These were then weighed and yield was expressed as $\mathrm{kg}$ per plant. For chemical analyses, twenty-four fruits from each treatment were selected from the last (fourth) cluster at the end of March and further divided into three batch samples. One batch of fruit was evaluated immediately after harvest and the other two batches were used for the assessment of storage performance. All the selected fruit were visually inspected prior to harvesting in order to make sure they were healthy and free of injuries, bruises and pathogen infestations. Moreover, the fruits affected by the physiological disorder "blossom-end rot" (BER) were also counted at each harvesting date.

\subsection{Analysis of the Drainage Solution}

Once a week, samples of the drainage solution were collected from every experimental plot. The samples were immediately taken to the laboratory and the $\mathrm{pH}$ and the EC were measured. The nitrates of the drainage solution were determined by means of a spectrophotometer according to the ultraviolet spectrophotometric screening method [34]. This technique is used for screening samples that have low organic matter contents. Measurement of UV absorption at $220 \mathrm{~nm}$ enables the rapid determination of $\mathrm{NO}_{3}$. Due to the fact that dissolved organic matter may also absorb at $220 \mathrm{~nm}$ and $\mathrm{NO}_{3}$ does not absorb at $275 \mathrm{~nm}$, a second measurement made at $275 \mathrm{~nm}$ and was sometimes used to correct the $\mathrm{NO}_{3}$ value.

\subsection{Mineral Composition Analysis}

After harvesting, leaf and fruit mineral content was determined. Sub-samples of 0.2-0.3 $\mathrm{g}$ were placed in the oven $\left(500{ }^{\circ} \mathrm{C}\right.$ for $\left.6 \mathrm{~h}\right)$ for ashing and were acid digested with $10 \mathrm{~mL} 2 \mathrm{~N} \mathrm{HCl}$. The content of $\mathrm{P}, \mathrm{K}, \mathrm{Ca}, \mathrm{Mg}, \mathrm{Na}$, aluminium (Al), Fe, $\mathrm{Zn}, \mathrm{Mn}$, $\mathrm{B}$ and $\mathrm{Cu}$ was determined with an atomic emission spectrometer (ICP-AES PSFO 2.0, Leeman Labs Inc., Hudson, NY, USA). The content of N was determined by using the Kjeldahl method (Kjeldahl nitrogen determination apparatus, Gerhardt Ltd., Brackley, UK). Data were expressed as $\mathrm{g} \mathrm{kg}^{-1}$ and $\mathrm{mg} \mathrm{kg}^{-1}$ dry weight for macro and micro-nutrients, respectively [17,35]. 


\subsection{Fruit Macroscopic Examination and Quality Analysis}

Fruit color was measured with CR-300 chromatometer (Minolta, Tokyo, Japan), with two measurements per fruit being taken on the equatorial axis. The values of $L^{*}, a^{*}$ and $b^{*}$ were measured (where: $L^{*}=$ lightness, $a^{*}=$ redness and $b^{*}=$ yellowness), and color indices (chroma, hue value, whitening index-WI and color index-CI) were computed [36]. Firmness was measured in one batch of the samples with a GY-2 penetrometer (Zhejiang Top Instrument, Hangzhou, China) equipped with a plunger of $3.5 \mathrm{~mm}$ in diameter. Fruits were probed randomly in two sides and data were expressed in Newton.

The other two batches of fruit were further used for postharvest performance, one batch of fruits was stored at room temperature at $25^{\circ} \mathrm{C}$, and the other group was stored in a fridge at $12{ }^{\circ} \mathrm{C}$ for 7 days. Following the 7 days of storage, the fruits were weighed again, evaluated macroscopically and measured for quality changes.

Fruit quality-related attributes, namely total soluble solids (TSS), titratable acidity (TA) and ascorbic acid (AA) content were measured [37]. TSS were measured with a digital refractometer (PR-100; ATAGO, Tokyo, Japan) and TSS content was expressed as ${ }^{\circ}$ Brix. For TA determination, tomato juice $(5 \mathrm{~mL})$ was diluted with distilled water to a final volume of $50 \mathrm{~mL}$, titrated with $0.1 \mathrm{~N} \mathrm{NaOH}$, in the presence of phenol-phtalein as an indicator, and expressed as citric acid percentage [37]. The ascorbic acid content was determined using the 2.6-Dichloroindophenol titrimetric method [38].

\subsection{Determination of Carotenoids and Total Phenolics}

Total phenolic composition was determined according to previous reports by the authors [33,37]. Briefly, methanolic extracts of blended tomato $(2.5 \mathrm{~g})$ following the addition of $1.5 \mathrm{~mL}$ of deionized water, $125 \mu \mathrm{L}$ of Folin-Ciocalteu's reagent (Merck Group, Darmstadt, Germany) and $1.25 \mathrm{~mL}$ of $7 \%(w / v)$ sodium carbonate and incubation for $90 \mathrm{~min}$ were measured at $755 \mathrm{~nm}$ (Specord 205, Analytik Jena, Jena, Germany). Results were expressed as gallic acid equivalents ( $\mu \mathrm{mol} \mathrm{GAE} \mathrm{g}^{-1}$ fresh weight).

Carotenoids (lycopene and $\beta$-carotene) content was measured according to the Nagata and Yamashita method with adjustments [39]. Briefly, $1 \mathrm{~g}$ of blended tomatoes was placed in $50 \mathrm{~mL}$ tubes and stored at $-20^{\circ} \mathrm{C}$. After de-freezing, $16 \mathrm{~mL}$ of acetone:hexane $4: 6(v: v)$ were added to each tube and shaken vigorously for phase separation. From each tube, an aliquot was taken with a syringe from the upper phase and measured with a spectrophotometer at $\lambda=663,645,505$ and $453 \mathrm{~nm}$, using as reference a solution of acetone:hexane (4:6) ratio. Carotenoids content was calculated according to the following Nagata and Yamashita [39] Equations (1) and (2):

Lycopene $\left(\mathrm{mg} 100 \mathrm{~mL}^{-1}\right.$ of extract $)=-0.0458 * \mathrm{~A} 663+0.204 * \mathrm{~A} 645+0.372 * \mathrm{~A} 505-0.0806 * \mathrm{~A} 453$.

$\beta$-carotene $\left(\mathrm{mg} 100 \mathrm{~mL}^{-1}\right.$ of extract $)=0.216 * \mathrm{~A} 663-1.22 * \mathrm{~A} 645-0.304 * \mathrm{~A} 505+0.452 * \mathrm{~A} 45$.

\subsection{Organoleptic Control}

The main idea was to study the effects of ammonium or salinity impacts on fruit quality (organoleptic test) immediately after harvest and to detect differences in the quality characteristics according to the treatments. Codded tomato samples from the six treatments (ammonium levels of 0.05, 0.10 and 0.15 ; salinity of low and high levels) were given as a blind test to a total of ten people (five men and five women) of various age groups and nationalities [37]. Participants were asked to rate the appearance, color, aroma, sweetness and texture of fruit on a scale of 1 to 5 (where 1 was for poor/soft/acidic/no, 2 for medium/little soft/less acidic/probably no, 3 for good/good/mild/sometimes, 4 for very good/little hard/little sweet/probably yes and 5 for excellent/hard/sweet/yes). Participants' preferences, the satisfaction index and the intention of the participants to buy the product were recorded too. The visual appearance evaluation was performed on intact fruit while the evaluation of aroma, texture and sweetness was performed on freshly cut 
fruit. All tests took place on lab benches with full light and without any disturbance during the testing procedure.

\subsection{Statistical Methods}

Statistical analysis was performed using the SPSS v22.0 (SPSS Inc., Chicago, IL, USA) program. Data were evaluated for normality before being subjected to analysis of variance (ANOVA). The significance of the differences between mean values was tested using the Duncan's multiple range test (DMRT) at $p \leq 0.05$, following one- and two-way ANOVA. For testing the panel's ratings, significant differences in rates were expressed in percentage values after a logarithmic transformation prior to analysis. Results were expressed as mean values \pm SE ( $n=8$ for plant growth/physiological/fruit quality and $n=4$ for chemical analyses).

\section{Results}

\subsection{Plant Growth and Physiological Parameters}

Table 1 presents the impacts of different ammonium to total nitrogen ratios as well as the different levels of salinity in the nutrient solution on plant growth-related attributes on hydroponically grown tomato plants. Two-way analysis of variance showed that $\mathrm{Nr}$ affected leaf photosynthesis and internal $\mathrm{CO}_{2}$ concentration $(p<0.05)$, whereas salinity affected tomato stem diameter, leaf photosynthesis and internal $\mathrm{CO}_{2}$ concentration $(p<0.05)$. The interaction of $\mathrm{Nr} \times$ salinity application affected fruit number $(p<0.001)$.

Table 1. The effect of salinity (Sal) levels (LS: low salinity of $0 \mathrm{mM} \mathrm{NaCl}$; HS: high salinity of $50 \mathrm{mM}$ $\mathrm{NaCl}$ ) and the application of $\mathrm{NH}_{4} / \mathrm{N}_{\text {total }}$ ratios $(\mathrm{Nr} 0.05, \mathrm{Nr} 0.10$ and $\mathrm{Nr} 0.15)$ on the plant growth, yield and physiological parameters of hydroponically grown tomato plants.

\begin{tabular}{|c|c|c|c|c|c|c|c|c|c|}
\hline & & Low Salinity & & & High Salinity & & & & \\
\hline & $\mathrm{Nr} 0.05+\mathrm{LS}$ & $\mathrm{Nr} 0.10+\mathrm{LS}$ & Nr0.15 + LS & $\mathrm{Nr} 0.05+\mathrm{HS}$ & $\mathrm{Nr} 0.10+\mathrm{HS}$ & Nr0.15 + HS & $\mathrm{Nr}$ & Sal & $\mathrm{Nr} \times \mathrm{Sal}$ \\
\hline Plant height (cm) & $180.00 \pm 6.87 \mathrm{a}$ & $183.37 \pm 5.16 \mathrm{a}$ & $170.62 \pm 5.18 \mathrm{ab}$ & $167.87 \pm 6.33 \mathrm{ab}$ & $149.00 \pm 7.92 \mathrm{c}$ & $160.85 \pm 3.46 \mathrm{bc}$ & ns & ns & ns \\
\hline Number of leaves & $22.50 \pm 0.70 \mathrm{a}$ & $21.62 \pm 0.59 a$ & $22.75 \pm 0.70 \mathrm{a}$ & $20.87 \pm 0.39 \mathrm{a}$ & $20.75 \pm 0.90 \mathrm{a}$ & $21.42 \pm 0.52 \mathrm{a}$ & ns & ns & ns \\
\hline Stem diameter $(\mathrm{mm})$ & $8.25 \pm 0.63 \mathrm{a}$ & $8.31 \pm 0.56 \mathrm{a}$ & $7.87 \pm 0.78 \mathrm{a}$ & $5.50 \pm 0.42 \mathrm{~b}$ & $5.12 \pm 0.45 \mathrm{~b}$ & $5.28 \pm 0.24 \mathrm{~b}$ & ns & * & ns \\
\hline Fruit number & $11.25 \pm 1.35 \mathrm{~b}$ & $15.33 \pm 1.66 \mathrm{a}$ & $12.14 \pm 1.10 \mathrm{ab}$ & $14.12 \pm 0.89 \mathrm{ab}$ & $11.71 \pm 1.04 \mathrm{ab}$ & $14.00 \pm 1.09 \mathrm{ab}$ & ns & ns & $* * *$ \\
\hline Fruit fresh weight (g) & $202.38 \pm 24.08$ a & $180.83 \pm 13.16 \mathrm{ab}$ & $188.16 \pm 8.74 \mathrm{a}$ & $125.83 \pm 2.96 \mathrm{c}$ & $144.64 \pm 7.51 \mathrm{bc}$ & $127.92 \pm 7.25 c$ & ns & ns & ns \\
\hline Yield (kg per plant) & $2.30 \pm 0.43 \mathrm{ab}$ & $2.71 \pm 0.24 \mathrm{a}$ & $2.29 \pm 0.26 \mathrm{ab}$ & $1.77 \pm 0.11 \mathrm{~b}$ & $1.67 \pm 0.13 \mathrm{~b}$ & $1.77 \pm 0.13 \mathrm{~b}$ & ns & ns & ns \\
\hline Chlorophyll a (mg g ${ }^{-1}$ ) & $14.60 \pm 1.86 \mathrm{a}$ & $9.18 \pm 1.62 \mathrm{a}$ & $13.91 \pm 0.96 \mathrm{a}$ & $13.14 \pm 2.27 \mathrm{a}$ & $14.52 \pm 2.58 \mathrm{a}$ & $11.79 \pm 2.00 \mathrm{a}$ & ns & ns & ns \\
\hline Chlorophyll b (mg g-1) & $6.23 \pm 0.80 \mathrm{a}$ & $3.61 \pm 0.57 \mathrm{a}$ & $5.52 \pm 0.51 \mathrm{a}$ & $6.30 \pm 1.10 \mathrm{a}$ & $5.85 \pm 0.96 \mathrm{a}$ & $5.39 \pm 1.24 \mathrm{a}$ & ns & ns & ns \\
\hline Total Chlorophylls a ( $\mathrm{mg} \mathrm{g}^{-1}$ ) & $20.82 \pm 2.63 \mathrm{a}$ & $12.80 \pm 2.19 \mathrm{a}$ & $19.43 \pm 1.39 \mathrm{a}$ & $19.42 \pm 3.32 \mathrm{a}$ & $20.37 \pm 3.54 \mathrm{a}$ & $17.17 \pm 3.24 \mathrm{a}$ & ns & ns & ns \\
\hline Photosynthetic rate $\left(\mu \mathrm{mol} \mathrm{m}^{-2} \mathrm{~s}^{-1}\right)$ & $11.46 \pm 1.99 \mathrm{~b}$ & $14.84 \pm 1.11 \mathrm{ab}$ & $17.82 \pm 1.14 \mathrm{a}$ & $17.52 \pm 2.14 \mathrm{a}$ & $18.17 \pm 1.58 \mathrm{a}$ & $15.60 \pm 0.85 \mathrm{ab}$ & * & * & ns \\
\hline Stomatal conductance $\left(\mu \mathrm{mol} \mathrm{m}^{-2} \mathrm{~s}^{-1}\right)$ & $0.611 \pm 0.054 \mathrm{a}$ & $0.687 \pm 0.052 \mathrm{a}$ & $0.683 \pm 0.122 \mathrm{a}$ & $0.529 \pm 0.055 \mathrm{a}$ & $0.660 \pm 0.040 \mathrm{a}$ & $0.455 \pm 0.157 \mathrm{a}$ & ns & ns & ns \\
\hline Internal $\mathrm{CO}_{2}\left(\mu \mathrm{mol} \mathrm{mol}^{-1}\right)$ & $280.66 \pm 31.59 b$ & $341.00 \pm 18.07 \mathrm{ab}$ & $385.00 \pm 30.09 \mathrm{ab}$ & $385.50 \pm 42.29 \mathrm{ab}$ & $395.50 \pm 31.33 \mathrm{a}$ & $313.00 \pm 35.79 \mathrm{ab}$ & * & * & ns \\
\hline
\end{tabular}

Results are expressed as means \pm SE $(n=8)$. Values in rows followed by the same Latin letter are not significantly different according to Duncan's multiple range test at $p \leq 0.05 . \mathrm{ns}^{*}{ }^{*}$ and ${ }^{* * *}$ indicate non-significant or significant differences at $p \leq 5 \%$ and $0.1 \%$, respectively, following two-way ANOVA.

A salinity of $50 \mathrm{mM} \mathrm{NaCl}$ at a medium ( $\mathrm{Nr} 0.10)$ and high ( $\mathrm{Nr} 0.15)$ ammonium to total nitrogen ratio decreased (up to $18.7 \%$ ) plant height in comparison to low salinity and low-medium $\mathrm{Nr}$ (Table 1). High salinity decreased stem diameter, fruit fresh weight and therefore crop yield in comparison to low saline-treated plants, while the fruit number produced remained unaffected. Plants grown under a low $\mathrm{Nr}$ ratio $(\mathrm{Nr} 0.05)$ revealed the lowest photosynthetic rates in comparison with the plants grown in $\mathrm{Nr} 0.15+\mathrm{LS}$, $\mathrm{Nr} 0.05+\mathrm{HS}$ and $\mathrm{Nr} 0.10+\mathrm{HS}$, and this counts as evidence for a higher internal $\mathrm{CO}_{2}$ concentration in the case of the latter ( $\mathrm{Nr} 0.10+\mathrm{HS})$.

Blossom end rot appeared above the third cluster and ranged from $7-9.4 \%$ of the fruits where salinity was applied, with $\mathrm{Nr} 0.05$ giving the lowest value, while the percentage of the fruits with blossom end rot was increased at higher $\mathrm{Nr}$ ratios.

\subsection{Drainage Solution and Leaf and Fruit Mineral Content}

Increasing $\mathrm{Nr}$ to 0.15 markedly suppressed the nutrient solution $\mathrm{pH}$ in the root environment of tomatoes as indicated by the values measured in the drainage water (Figure S2). This effect was more profound after the 13th of December and until the middle of February due to the low air temperature and light intensity on that period. Even an $\mathrm{Nr}$ of 0.15 
resulted in $\mathrm{pH}$ values below 5.0 during that season. Conversely, when $\mathrm{Nr}$ was as low as 0.05 , the $\mathrm{pH}$ in the root zone was raised to values above 7.0 during the winter. However, salinity did not have any effect on the drainage solution $\mathrm{pH}$, but salinity increased the EC of the drainage solution (varying from 8 to $12 \mathrm{mS} \mathrm{cm}^{-1}$ ), with a more pronounced increase at the lower $\mathrm{Nr}$ (Figure S2). Increased salinity and $\mathrm{Nr}$ resulted in decreased nitrate accumulation in the drainage nutrient solution (Figure S2).

The impacts of salinity and/or ammonium to total nitrogen levels on mineral content in tomato leaves and fruits is presented in Table 2. Two-way ANOVA revealed that $\mathrm{Nr}$ significantly affected the content of $p(p<0.05)$ in leaves, while salinity significantly affected the content of $\mathrm{Mg}$ and $\mathrm{Na}(p<0.001)$, specifically $\mathrm{K}, \mathrm{P}$ and $\mathrm{B}(p<0.01)$ and $\mathrm{N}$ and $\mathrm{Zn}$ $(p<0.05)$, in leaves. The interaction of $\mathrm{Nr} \times$ salinity affected $\mathrm{N}$ content $(p<0.001)$ in leaves. In fruit, salinity significantly affected $\mathrm{Na}(p<0.01)$, specifically $\mathrm{N}, \mathrm{K}$, and $\mathrm{Mn}(p<0.05)$, whereas the interaction of $\mathrm{Nr} \times$ salinity affected Mn content $(p<0.05)$. No changes on fruit mineral content were found in terms of the different $\mathrm{Nr}$ applications (Table 2).

Table 2. The effect of salinity (Sal) levels (LS: low salinity of $0 \mathrm{mM} \mathrm{NaCl}$; HS: high salinity of $50 \mathrm{mM}$ $\mathrm{NaCl})$ and the application of $\mathrm{NH}_{4} / \mathrm{N}_{\text {total }}$ ratios $(\mathrm{Nr} 0.05, \mathrm{Nr} 0.10$ and $\mathrm{Nr} 0.15)$ on mineral content of tomato leaves and fruit of hydroponically grown tomato plants.

\begin{tabular}{|c|c|c|c|c|c|c|c|c|c|}
\hline & & Low Salinity & & & High Salinity & & & & \\
\hline Leaves & Nr0.05 + LS & $\mathrm{Nr0.10}+$ LS & Nr0.15 + LS & Nr0.05 + HS & $\mathrm{Nr} 0.10+\mathrm{HS}$ & Nr0.15 + HS & $\mathrm{Nr}$ & Sal & $\mathrm{Nr} \times \mathrm{Sal}$ \\
\hline $\mathrm{N}\left(\mathrm{g} \mathrm{kg}^{-1}\right)$ & $31.17 \pm 0.41 \mathrm{a}$ & $26.82 \pm 1.02 b$ & $27.10 \pm 0.49 \mathrm{~b}$ & $28.77 \pm 0.72 b$ & $33.35 \pm 0.63 a$ & $33.50 \pm 1.03 \mathrm{a}$ & ns & $*$ & $* * *$ \\
\hline $\mathrm{K}\left(\mathrm{g} \mathrm{kg}^{-1}\right)$ & $33.17 \pm 1.45 \mathrm{a}$ & $32.11 \pm 1.55 \mathrm{a}$ & $34.74 \pm 1.00 \mathrm{a}$ & $24.43 \pm 1.51 \mathrm{~b}$ & $21.70 \pm 0.67 \mathrm{~b}$ & $16.32 \pm 1.72 \mathrm{c}$ & ns & $* *$ & ns \\
\hline $\mathrm{Ca}\left(\mathrm{g} \mathrm{kg}^{-1}\right)$ & $26.46 \pm 12.05 \mathrm{a}$ & $23.63 \pm 7.21 \mathrm{a}$ & $19.11 \pm 2.19 a$ & $23.71 \pm 4.66 \mathrm{a}$ & $29.76 \pm 5.81 \mathrm{a}$ & $32.92 \pm 10.67 \mathrm{a}$ & ns & ns & ns \\
\hline$P\left(\mathrm{~g} \mathrm{~kg}^{-1}\right)$ & $3.02 \pm 0.25 \mathrm{a}$ & $2.86 \pm 0.45 a$ & $3.90 \pm 0.45 \mathrm{a}$ & $5.31 \pm 1.09 \mathrm{a}$ & $4.05 \pm 0.44 \mathrm{a}$ & $4.91 \pm 1.66 \mathrm{a}$ & $*$ & $* *$ & ns \\
\hline $\operatorname{Mg}\left(\mathrm{g} \mathrm{kg}^{-1}\right)$ & $3.98 \pm 0.33 c$ & $3.93 \pm 0.34 c$ & $4.25 \pm 0.26 \mathrm{c}$ & $8.17 \pm 0.62 b$ & $7.51 \pm 0.88 b$ & $10.85 \pm 1.36 \mathrm{a}$ & ns & $* * *$ & ns \\
\hline $\mathrm{Na}\left(\mathrm{g} \mathrm{kg}^{-1}\right)$ & $2.08 \pm 0.18 \mathrm{c}$ & $2.23 \pm 0.15 c$ & $2.41 \pm 0.40 \mathrm{c}$ & $10.04 \pm 1.10 \mathrm{~b}$ & $8.55 \pm 1.19 b$ & $13.95 \pm 2.02 \mathrm{a}$ & ns & $* * *$ & ns \\
\hline $\mathrm{Fe}\left(\mathrm{mg} \mathrm{kg}^{-1}\right)$ & $80.17 \pm 6.05 \mathrm{ab}$ & $71.49 \pm 3.26 \mathrm{~b}$ & $73.36 \pm 3.53 \mathrm{ab}$ & $76.98 \pm 2.13 \mathrm{ab}$ & $78.38 \pm 5.26 \mathrm{ab}$ & $89.22 \pm 7.92 \mathrm{a}$ & ns & ns & ns \\
\hline $\mathrm{Zn}\left(\mathrm{mg} \mathrm{kg}^{-1}\right)$ & $4.87 \pm 0.82 c$ & $5.73 \pm 2.03 c$ & $6.27 \pm 2.67 c$ & $17.69 \pm 1.96 \mathrm{~b}$ & $16.89 \pm 3.83 b$ & $37.53 \pm 8.25 \mathrm{a}$ & ns & $*$ & ns \\
\hline $\mathrm{Cu}\left(\mathrm{mg} \mathrm{kg}^{-1}\right)$ & $1.04 \pm 0.24 \mathrm{a}$ & $1.35 \pm 0.46 \mathrm{a}$ & $0.55 \pm 0.21 \mathrm{a}$ & $2.05 \pm 0.54 \mathrm{a}$ & $1.48 \pm 0.49 \mathrm{a}$ & $3.98 \pm 0.83 \mathrm{a}$ & ns & ns & ns \\
\hline $\operatorname{Mn}\left(\mathrm{mg} \mathrm{kg}^{-1}\right)$ & $24.21 \pm 2.36 \mathrm{~b}$ & $29.77 \pm 7.82 \mathrm{ab}$ & $40.97 \pm 13.22 \mathrm{ab}$ & $47.28 \pm 10.47 \mathrm{ab}$ & $51.37 \pm 7.97 \mathrm{ab}$ & $79.98 \pm 33.47 \mathrm{a}$ & ns & ns & ns \\
\hline$B\left(\mathrm{mg} \mathrm{kg}^{-1}\right)$ & $72.47 \pm 3.62 \mathrm{ab}$ & $63.16 \pm 7.69 \mathrm{~b}$ & $69.34 \pm 3.74 \mathrm{ab}$ & $84.17 \pm 5.56 \mathrm{ab}$ & $89.81 \pm 5.80 \mathrm{a}$ & $79.52 \pm 12.57 \mathrm{ab}$ & ns & $* *$ & ns \\
\hline \multicolumn{10}{|l|}{ Fruits } \\
\hline$N\left(\mathrm{~g} \mathrm{~kg}^{-1}\right)$ & $29.90 \pm 7.01 \mathrm{a}$ & $29.35 \pm 5.28 \mathrm{a}$ & $19.12 \pm 1.61 \mathrm{ab}$ & $16.87 \pm 0.95 b$ & $16.75 \pm 0.72 b$ & $18.55 \pm 1.97 \mathrm{ab}$ & ns & * & ns \\
\hline $\mathrm{K}_{\left(\mathrm{g} \mathrm{kg}^{-1}\right)}$ & $27.60 \pm 1.56 \mathrm{a}$ & $24.10 \pm 2.82 \mathrm{ab}$ & $24.86 \pm 1.01 \mathrm{ab}$ & $21.22 \pm 1.90 \mathrm{bc}$ & $19.77 \pm 1.68 \mathrm{bc}$ & $16.95 \pm 1.72 c$ & ns & * & ns \\
\hline $\mathrm{Ca}\left(\mathrm{g} \mathrm{kg}^{-1}\right)$ & $2.65 \pm 0.30 \mathrm{a}$ & $1.70 \pm 0.11 \mathrm{~b}$ & $1.62 \pm 0.02 \mathrm{~b}$ & $1.47 \pm 0.08 \mathrm{~b}$ & $1.54 \pm 0.09 \mathrm{~b}$ & $1.32 \pm 0.05 \mathrm{~b}$ & ns & ns & ns \\
\hline$p\left(\mathrm{~g} \mathrm{~kg}^{-1}\right)$ & $1.86 \pm 0.18 \mathrm{a}$ & $1.26 \pm 0.08 \mathrm{~b}$ & $1.56 \pm 0.27 \mathrm{ab}$ & $1.13 \pm 0.22 b$ & $1.12 \pm 0.15 \mathrm{~b}$ & $1.15 \pm 0.15 \mathrm{~b}$ & ns & $\mathrm{ns}$ & ns \\
\hline $\operatorname{Mg}\left(\mathrm{g} \mathrm{kg}^{-1}\right)$ & $0.73 \pm 0.07 \mathrm{a}$ & $0.55 \pm 0.01 \mathrm{ab}$ & $0.70 \pm 0.14 \mathrm{a}$ & $0.44 \pm 0.06 \mathrm{~b}$ & $0.49 \pm 0.06 \mathrm{ab}$ & $0.56 \pm 0.03 \mathrm{ab}$ & ns & ns & ns \\
\hline $\mathrm{Na}\left(\mathrm{g} \mathrm{kg}^{-1}\right)$ & $0.77 \pm 0.08 \mathrm{~b}$ & $0.45 \pm 0.09 \mathrm{~b}$ & $0.57 \pm 0.09 \mathrm{~b}$ & $2.10 \pm 0.39 \mathrm{a}$ & $2.17 \pm 0.15 \mathrm{a}$ & $1.86 \pm 0.29 \mathrm{a}$ & ns & $* *$ & ns \\
\hline $\mathrm{Al}\left(\mathrm{g} \mathrm{kg}^{-1}\right)$ & $1.36 \pm 0.05 \mathrm{a}$ & $0.95 \pm 0.20 \mathrm{ab}$ & $0.71 \pm 0.20 \mathrm{~b}$ & $1.11 \pm 0.09 \mathrm{ab}$ & $1.08 \pm 0.10 \mathrm{ab}$ & $0.82 \pm 0.12 \mathrm{~b}$ & $*$ & ns & ns \\
\hline $\mathrm{Fe}\left(\mathrm{mg} \mathrm{kg}^{-1}\right)$ & $47.72 \pm 6.66 \mathrm{ab}$ & $48.60 \pm 2.35 \mathrm{ab}$ & $67.53 \pm 23.42 \mathrm{a}$ & $31.72 \pm 3.36 \mathrm{~b}$ & $28.10 \pm 3.37 b$ & $29.22 \pm 2.19 b$ & ns & ns & ns \\
\hline $\mathrm{Zn}\left(\mathrm{mg} \mathrm{kg}^{-1}\right)$ & $3.85 \pm 0.46 \mathrm{a}$ & $7.53 \pm 2.77 \mathrm{a}$ & $6.38 \pm 3.27 \mathrm{a}$ & $3.10 \pm 1.23 \mathrm{a}$ & $1.05 \pm 0.38 \mathrm{a}$ & $3.53 \pm 1.62 \mathrm{a}$ & ns & ns & ns \\
\hline $\mathrm{Cu}\left(\mathrm{mg} \mathrm{kg}^{-1}\right)$ & $1.88 \pm 0.36 \mathrm{a}$ & $1.57 \pm 0.77 \mathrm{ab}$ & $0.55 \pm 0.20 \mathrm{ab}$ & nd & nd & $0.28 \pm 0.01 b$ & ns & ns & ns \\
\hline $\operatorname{Mn}\left(\mathrm{mg} \mathrm{kg}^{-1}\right)$ & $14.32 \pm 1.74 \mathrm{a}$ & $4.88 \pm 0.18 b$ & $7.57 \pm 0.91 \mathrm{~b}$ & $4.01 \pm 1.61 \mathrm{~b}$ & $4.57 \pm 3.13 b$ & $3.23 \pm 0.36 b$ & ns & $*$ & $*$ \\
\hline$B\left(\mathrm{mg} \mathrm{kg}^{-1}\right)$ & $18.20 \pm 3.36 \mathrm{a}$ & $14.46 \pm 3.83 \mathrm{a}$ & $18.19 \pm 9.15 \mathrm{a}$ & $12.07 \pm 3.57 \mathrm{a}$ & $13.36 \pm 3.51 \mathrm{a}$ & $8.39 \pm 1.59 \mathrm{a}$ & ns & ns & $\mathrm{ns}$ \\
\hline
\end{tabular}

Results are expressed as mean values \pm SE $(n=4)$. Values in rows followed by the same Latin letter are not significantly different according to Duncan's multiple range test at $p \leq 0.05$; nd-not detectable. ns, * $* *$ and ${ }^{* *}$ indicate non-significant or significant differences at $p \leq 5 \%, 1 \%$ and $0.1 \%$, respectively, following two-way ANOVA.

In leaves, $\mathrm{N}$ content increased at low $\mathrm{Nr}$ levels (i.e., $\mathrm{Nr0} 05+\mathrm{LS}$ ), but decreased at $\geq \mathrm{Nr} 0.10+$ LS, while the opposite effects were observed in saline-treated plants, with $\mathrm{N}$ content increasing at $\geq \mathrm{Nr0} .10+\mathrm{HS}$ (Table 2). In contrast, leaf K content decreased (up to $53 \%$ ) in saline-treated plants, independently of the $\mathrm{Nr}$ levels. The content of $\mathrm{Mg}, \mathrm{Na}$ and $\mathrm{Zn}$ was increased in saline-treated plants, and the levels were more pronounced at the high $\mathrm{Nr}$ ratio of $0.15(\mathrm{Nr} 0.15+\mathrm{HS})$. The content of Fe and $\mathrm{Mn}$ was greater as a result of the $\mathrm{Nr} 0.15$ + HS treatment, while the greater B content was found as a result of the $\mathrm{Nr} 0.10+\mathrm{HS}$ treatment. No differences were observed in leaf $\mathrm{Ca}, \mathrm{P}$ and $\mathrm{Cu}$ content as averaged in $25.93 \mathrm{~g} \mathrm{~kg}^{-1}, 4.01 \mathrm{~g} \mathrm{~kg}^{-1}$ and $1.74 \mathrm{mg} \mathrm{kg}^{-1}$, respectively.

In the fruits, the content of $\mathrm{N}, \mathrm{K}, \mathrm{Ca}, \mathrm{P}$ and $\mathrm{Mg}$ was increased in non-saline treated plants and at a low $\mathrm{Nr}$ ratio, while $\mathrm{Mg}$ was also accumulated in case of a higher $\mathrm{Nr}$ ratio in non-saline treated tomatoes (Table 2). Indeed, $\mathrm{Na}$ was accumulated in saline treated plants, independently of the $\mathrm{Nr}$ ratio. Copper and $\mathrm{Mn}$ content was greatest at low $\mathrm{Nr}$ ratios in non-saline treated plants. Iron content was increased at Nr0.15 + LS but decreased 
in saline-treated plants. Both $\mathrm{Zn}$ and B remained unchanged in all examined treatments (Table 2).

\subsection{Quality Parameters at Harvested Fruits}

Harvested fruits were analyzed for quality related attributes. The analysis of variance of the fruit quality data revealed that fruit dry matter content, TSS, Vitamin C content $(p<0.001)$, citric acid content, color index $(p<0.01)$, fruit firmness, color $b^{*}$ and Hue $(p<0.05)$ were all influenced by salinity. The $\mathrm{Nr}$ ratio did not affect fruit quality parameters, while the interaction $\mathrm{Nr} \times$ salinity significantly affected the color index $(p<0.01)$, Hue and whitening index $(p<0.05)$, as presented in Table 3 .

Table 3. The effect of salinity (Sal) levels (LS: low salinity of $0 \mathrm{mM} \mathrm{NaCl}$; HS: high salinity of 50 $\mathrm{mM} \mathrm{NaCl})$ and the application of $\mathrm{NH}_{4} / \mathrm{N}_{\text {total }}$ ratios $(\mathrm{Nr} 0.05, \mathrm{Nr} 0.10$, and $\mathrm{Nr} 0.15)$ on the tomato fruit quality related attributes of hydroponically grown tomato plants.

\begin{tabular}{|c|c|c|c|c|c|c|c|c|c|}
\hline & & Low Salinity & & & High Salinity & & & & \\
\hline & $\mathrm{Nr} 0.05+\mathrm{LS}$ & Nr0.10 + LS & Nr0.15 + LS & $\mathrm{Nr} 0.05+\mathrm{HS}$ & $\mathrm{Nr} 0.10+\mathrm{HS}$ & Nr0.15 + HS & $\mathrm{Nr}$ & Sal & $\mathrm{Nr} \times \mathrm{Sal}$ \\
\hline Dry matter content $(\%)$ & $4.48 \pm 0.32 b$ & $5.02 \pm 0.14 \mathrm{~b}$ & $4.78 \pm 0.12 b$ & $6.73 \pm 0.10 \mathrm{ab}$ & $8.98 \pm 2.02 \mathrm{a}$ & $6.92 \pm 0.28 \mathrm{ab}$ & ns & $* * *$ & ns \\
\hline Firmness (N) & $5.18 \pm 0.35 c$ & $4.77 \pm 0.20 c$ & $5.58 \pm 0.31 \mathrm{bc}$ & $5.31 \pm 0.36 c$ & $6.31 \pm 0.30 \mathrm{ab}$ & $6.75 \pm 0.26 \mathrm{a}$ & ns & $*$ & $\mathrm{~ns}$ \\
\hline TSS $\left({ }^{\circ}\right.$ Brix $)$ & $4.00 \pm 0.23 b$ & $3.84 \pm 0.16 \mathrm{~b}$ & $4.12 \pm 0.14 \mathrm{~b}$ & $5.46 \pm 0.20 \mathrm{a}$ & $5.76 \pm 0.21 \mathrm{a}$ & $5.22 \pm 0.25 \mathrm{a}$ & ns & $* * *$ & ns \\
\hline Citric acid (\%) & $0.21 \pm 0.01 \mathrm{~b}$ & $0.20 \pm 0.02 \mathrm{~b}$ & $0.21 \pm 0.01 \mathrm{~b}$ & $0.27 \pm 0.03 \mathrm{a}$ & $0.24 \pm 0.02 \mathrm{ab}$ & $0.27 \pm 0.01 \mathrm{a}$ & ns & $* *$ & ns \\
\hline Ripening (TSS/TA) & $18.95 \pm 1.17 \mathrm{a}$ & $20.00 \pm 2.53 \mathrm{a}$ & $20.58 \pm 2.36 \mathrm{a}$ & $20.89 \pm 2.57 \mathrm{a}$ & $24.12 \pm 2.07 \mathrm{a}$ & $19.26 \pm 1.62 \mathrm{a}$ & ns & ns & ns \\
\hline Color ( $L^{*}$ value $)$ & $35.70 \pm 0.83 \mathrm{a}$ & $34.30 \pm 0.44 \mathrm{ab}$ & $34.77 \pm 0.68 \mathrm{ab}$ & $33.79 \pm 0.23 \mathrm{~b}$ & $33.83 \pm 0.31 \mathrm{~b}$ & $33.89 \pm 0.33 \mathrm{~b}$ & ns & ns & ns \\
\hline Color ( $a^{*}$ value $)$ & $18.65 \pm 1.53 \mathrm{a}$ & $20.08 \pm 1.07 \mathrm{a}$ & $20.26 \pm 1.26 \mathrm{a}$ & $21.02 \pm 0.64 \mathrm{a}$ & $18.39 \pm 0.53 \mathrm{a}$ & $20.55 \pm 0.99 a$ & ns & ns & ns \\
\hline Color ( $b^{*}$ value) & $12.75 \pm 0.66 \mathrm{a}$ & $12.26 \pm 0.62 \mathrm{a}$ & $12.61 \pm 0.36 \mathrm{a}$ & $11.74 \pm 0.33 \mathrm{a}$ & $11.45 \pm 0.24 \mathrm{a}$ & $11.89 \pm 0.24 \mathrm{a}$ & ns & $*$ & ns \\
\hline Chroma & $22.61 \pm 1.60 \mathrm{a}$ & $23.53 \pm 1.22 \mathrm{a}$ & $23.89 \pm 1.21 \mathrm{a}$ & $24.08 \pm 0.64 \mathrm{a}$ & $21.67 \pm 0.51 \mathrm{a}$ & $23.75 \pm 0.97 \mathrm{a}$ & ns & ns & ns \\
\hline Hue & $0.60 \pm 0.01 \mathrm{a}$ & $0.54 \pm 0.00 \mathrm{bc}$ & $0.56 \pm 0.02 \mathrm{ab}$ & $0.51 \pm 0.01 \mathrm{c}$ & $0.55 \pm 0.01 \mathrm{abc}$ & $0.53 \pm 0.01 \mathrm{bc}$ & ns & $*$ & * \\
\hline Whitening index & $31.74 \pm 0.42 \mathrm{a}$ & $30.17 \pm 0.32 \mathrm{~b}$ & $30.48 \pm 0.50 \mathrm{~b}$ & $29.53 \pm 0.33 b$ & $30.36 \pm 0.40 \mathrm{~b}$ & $29.72 \pm 0.33 \mathrm{~b}$ & ns & ns & * \\
\hline Color index & $40.82 \pm 1.19 \mathrm{c}$ & $47.82 \pm 1.38 \mathrm{~b}$ & $46.21 \pm 2.25 \mathrm{~b}$ & $53.11 \pm 1.72 \mathrm{a}$ & $47.56 \pm 1.65 \mathrm{~b}$ & $50.94 \pm 1.71 \mathrm{~b}$ & ns & $* *$ & ** \\
\hline Vitamin C (\%) & $0.59 \pm 0.08 \mathrm{bc}$ & $0.52 \pm 0.05 c$ & $0.74 \pm 0.07 \mathrm{bc}$ & $0.92 \pm 0.13 \mathrm{ab}$ & $1.15 \pm 0.18 \mathrm{a}$ & $1.14 \pm 0.11 \mathrm{a}$ & ns & $* * *$ & ns \\
\hline Lycopene $\left(\mathrm{mg} \mathrm{g}^{-1} \mathrm{Fw}\right)$ & $0.054 \pm 0.014 \mathrm{a}$ & $0.056 \pm 0.009 \mathrm{a}$ & $0.039 \pm 0.009 \mathrm{a}$ & $0.073 \pm 0.013 \mathrm{a}$ & $0.071 \pm 0.013 \mathrm{a}$ & $0.054 \pm 0.005 \mathrm{a}$ & ns & ns & ns \\
\hline$\beta$-carotene $\left(\mathrm{mg} \mathrm{g}^{-1} \mathrm{Fw}\right)$ & $0.052 \pm 0.007 \mathrm{a}$ & $0.044 \pm 0.004 \mathrm{a}$ & $0.038 \pm 0.010 \mathrm{a}$ & $0.041 \pm 0.004 \mathrm{a}$ & $0.049 \pm 0.004 \mathrm{a}$ & $0.049 \pm 0.005 \mathrm{a}$ & ns & ns & ns \\
\hline Total phenols ( $\left.\mu \mathrm{mol} \mathrm{GAE} \mathrm{g}^{-1} \mathrm{Fw}\right)$ & $0.35 \pm 0.02 \mathrm{ab}$ & $0.29 \pm 0.02 \mathrm{ab}$ & $0.28 \pm 0.01 \mathrm{~b}$ & $0.38 \pm 0.04 \mathrm{a}$ & $0.35 \pm 0.02 \mathrm{ab}$ & $0.32 \pm 0.01 \mathrm{ab}$ & ns & ns & ns \\
\hline
\end{tabular}

Results are expressed as means \pm SE $(n=8)$. Values in rows followed by the same Latin letter are not significantly different according to Duncan's multiple range test at $p \leq 0.05$. ns, ${ }^{*}, * *$ and ${ }^{* * *}$ indicate non-significant or significant differences at $p \leq 5 \%, 1 \%$ and $0.1 \%$, respectively, following two-way ANOVA.

The content of TSS in tomato fruit was significantly higher in plants grown under salinity $(50 \mathrm{mM} \mathrm{NaCl})$, regardless of the $\mathrm{Nr}$ ratio, as shown in Table 3 . Citric acid content was lower in non-saline grown plants. Fruit firmness was the greatest in the saline-treated plants with a high $\mathrm{Nr}$ ratio $(\mathrm{Nr} 0.15+\mathrm{HS})$, while a low $\mathrm{Nr}$ ratio resulted in decreased firmness. Plants grown in saline conditions produced fruits with increased dry matter content, which significantly differed at $\mathrm{Nr} 0.10+$ HS compared to the fruits harvested from the non-saline grown plants. Vitamin $C$ content in tomato fruit was increased at a salinity of $50 \mathrm{mM} \mathrm{NaCl}$ at $\geq \mathrm{Nr} 0.10$ (i.e., $\mathrm{Nr} 0.10+\mathrm{HS}$ and $\mathrm{Nr} 0.15+\mathrm{HS}$ ) in comparison to the fruits harvested from the non-saline grown plants.

Fruit lightness was decreased in saline-treated plants compared to the non-saline grown plants (Table 3). A higher $L^{*}$ value in fruit was found in the case of the low $\mathrm{Nr}$ ratio in non-saline grown plants, which resulted in the Hue and whitening index being increased and the color index being decreased. The color $a^{*}$ and $b^{*}$ values, and therefore the fruit chroma, was unchanged in the examined $\mathrm{NaCl}$ and $\mathrm{Nr}$ applications.

The total phenols content increased at the $\mathrm{Nr} 0.05+\mathrm{HS}$, whereas the lycopene and $\beta$-carotene content was the same for all the treatments, with an average of 0.058 and $0.046 \mathrm{mg} \mathrm{g}^{-1}$ fresh weight for lycopene and $\beta$-carotene, respectively (Table 3 ).

\subsection{Organoleptic Test}

Sensory evaluation took place through a panel test of the examined samples at the day of harvest for fruit harvested within a 5-days harvesting period (Table 4). Considering the salinity effect, all testers were able to determine variations between the tested samples. Fifty percent of the testers preferred the fruits that were harvested from saline-grown plants, $40 \%$ of the testers preferred fruits harvested from non-saline grown plants, whereas only $10 \%$ of the participants preferred none of the examined samples (data not presented). Higher aroma and sweetness scores were found in fruit subjected to salinity, while fruit 
appearance, color, texture, overall satisfaction and marketability did not differ significantly with a salinity of $50 \mathrm{mM} \mathrm{NaCl}$ (despite the increased scores in saline treated tomatoes compared to the non-saline treated fruit).

Table 4. Quantitative analysis, expressed as percentage values (\%) of quality attributes in relation to salinity levels ( 0 and $50 \mathrm{mM} \mathrm{NaCl})$ and/or the application of $\mathrm{NH}_{4} / \mathrm{N}_{\text {total }}$ ratios $(\mathrm{Nr} 0.05, \mathrm{Nr} 0.10$ and $\mathrm{Nr} 0.15$ ) on hydroponically grown tomatoes. Assessments were reported by 10 panelists per treatment.

\begin{tabular}{|c|c|c|c|c|c|c|c|c|}
\hline Treatment & & Appearance & Color & Aroma & Texture & Sweetness & $\begin{array}{c}\text { Overall } \\
\text { Satisfaction }\end{array}$ & Marketability \\
\hline $\mathrm{NH}_{4} / \mathrm{N}_{\text {total }}$ ratios & Nr0.05 & $66.00 \pm 4.26 \mathrm{a}$ & $64.00 \pm 2.66 \mathrm{~b}$ & $62.00 \pm 6.28 \mathrm{a}$ & $84.00 \pm 5.81 \mathrm{a}$ & $58.00 \pm 4.66 \mathrm{a}$ & $64.00 \pm 4.98 \mathrm{a}$ & $68.00 \pm 4.42 \mathrm{a}$ \\
\hline \multirow{4}{*}{ Salinity } & $\mathrm{Nr} 0.10$ & $78.00 \pm 4.66 \mathrm{a}$ & $76.00 \pm 4.00 \mathrm{a}$ & $76.00 \pm 4.98 \mathrm{a}$ & $82.00 \pm 6.28 \mathrm{a}$ & $70.00 \pm 5.37 \mathrm{a}$ & $74.00 \pm 3.05 \mathrm{a}$ & $80.00 \pm 5.16 a$ \\
\hline & Nr0.15 & $76.00 \pm 4.00 \mathrm{a}$ & $75.00 \pm 3.33 \mathrm{a}$ & $70.00 \pm 6.14 \mathrm{a}$ & $84.00 \pm 4.98 \mathrm{a}$ & $66.00 \pm 4.26 \mathrm{a}$ & $69.33 \pm 2.99 a$ & $74.00 \pm 5.20 \mathrm{a}$ \\
\hline & $0 \mathrm{mM} \mathrm{NaCl}$ & $66.00 \pm 3.05 \mathrm{a}$ & $78.00 \pm 3.59 \mathrm{a}$ & $56.00 \pm 4.98 \mathrm{~b}$ & $70.00 \pm 6.14 \mathrm{a}$ & $74.00 \pm 3.05 b$ & $66.00 \pm 4.26 \mathrm{a}$ & $74.00 \pm 5.20 \mathrm{a}$ \\
\hline & $50 \mathrm{mM} \mathrm{NaCl}$ & $66.00 \pm 5.02 \mathrm{a}$ & $68.00 \pm 5.33 a$ & $76.00 \pm 5.81 \mathrm{a}$ & $82.00 \pm 7.57 \mathrm{a}$ & $82.00 \pm 2.53 \mathrm{a}$ & $70.00 \pm 6.14 a$ & $76.00 \pm 6.53 \mathrm{a}$ \\
\hline
\end{tabular}

Results are expressed as mean \pm SE $(n=10)$. In each treatment, values in columns followed by the same Latin letter are not significantly different according to Duncan's multiple range test at $p \leq 0.05$.

Considering the $\mathrm{Nr}$ effect, $90 \%$ of the testers could determine differences between samples. Fifty percent of the testers preferred the fruits that were harvested from high $\mathrm{Nr}$ ratio (Nr0.15) treated plants, 30\% of the testers preferred fruits harvested from $\mathrm{Nr} 0.10$ treated plants and $20 \%$ of the testers preferred fruits harvested from $\mathrm{Nr} 0.05$ treated plants (data not presented). The greatest scores for fruit color were found in the cases of Nr0.10 and Nr0.15 treated plants, while lower color scores were found for the $\mathrm{Nr} 0.05$ treatment. All the other fruit quality related attributes revealed similar scores by the testers.

\subsection{Postharvest Fruit Quality Attributes}

Harvested fruits from plants grown under a salinity of 0 or $50 \mathrm{mM} \mathrm{NaCl}$ and/or an ammonium to total nitrogen ratio of $\mathrm{Nr} 0.05, \mathrm{Nr} 0.10$ or $\mathrm{Nr} 0.15$ were stored for 7 days at $12{ }^{\circ} \mathrm{C}$ and $25^{\circ} \mathrm{C}$, reflecting storage conditions in packinghouse or shelf-life conditions. At $12{ }^{\circ} \mathrm{C}$, fruit firmness was maintained for the $\mathrm{Nr} 0.15+\mathrm{HS}$ plants in comparison to the $\mathrm{Nr} 0.05$ + LS ones (Figure 1B). A salinity of $50 \mathrm{mM} \mathrm{NaCl}$ increased fruit TSS, independently of the different $\mathrm{Nr}$ (Figure 1C), while the fruit weight loss, TA and ripening index (TSS/TA) were not affected by neither salinity nor $\mathrm{Nr}$ (Figure $1 \mathrm{~A}, \mathrm{D}, \mathrm{E}$ ). At $25^{\circ} \mathrm{C}$, fruit firmness was maintained for the saline-treated plants at all Nr levels as well as for the $\mathrm{Nr} 0.10+\mathrm{LS}$ treatment in comparison to Nr0.05 + LS (Figure 1B). Fruit weight loss decreased for the $\mathrm{Nr} 0.05$ + HS plants compared to the Nr0.15 + LS ones (Figure 1A). Similar to the results at $12{ }^{\circ} \mathrm{C}$, at $25^{\circ} \mathrm{C}$, fruit TSS was increased in saline-treated plants, independent of the different $\mathrm{Nr}$ ratios (Figure 1C). Fruit titratable acidity was increased at $\geq \mathrm{Nr} 0.10$ in saline-treated plants compared to the non-saline treated plants (Figure 1D). The highest ripening index was observed for the Nr0.05 + HS plants in comparison to the other treatments (Figure 1E).

Fruit color attributes were more affected in commodities stored at $12{ }^{\circ} \mathrm{C}$ than in commodities stored at $25{ }^{\circ} \mathrm{C}$ (Figure 2). At $12{ }^{\circ} \mathrm{C}, \mathrm{Nr} 0.15+\mathrm{LS}$ treatment revealed fruits with increased lightness $\left(L^{*}\right)$, redness $\left(a^{*}\right)$, yellowness $\left(b^{*}\right)$ and chroma (Figure $\left.2 \mathrm{~A}-\mathrm{D}\right)$. The greatest Hue value was found for the Nr0.05 + LS plants, the greatest WI was found for $\mathrm{Nr} 0.10$ + LS plants, while the greatest color index was observed for $\mathrm{Nr} 0.05+$ HS plants (Figure 2E-G). At $25^{\circ} \mathrm{C}$, the Nr0.05 + LS treatment revealed fruits with increased lightness $\left(L^{*}\right)$, yellowness $\left(b^{*}\right)$ and Hue values (Figure $2 \mathrm{~A}-\mathrm{E}$ ) but with a lower color index (Figure 2G). Indeed, no differences were found in color $a^{*}$, chroma and WI among the saline and/or $\mathrm{Nr}$ treatments at $25^{\circ} \mathrm{C}$ storage conditions (Figure 2B,D,F). 

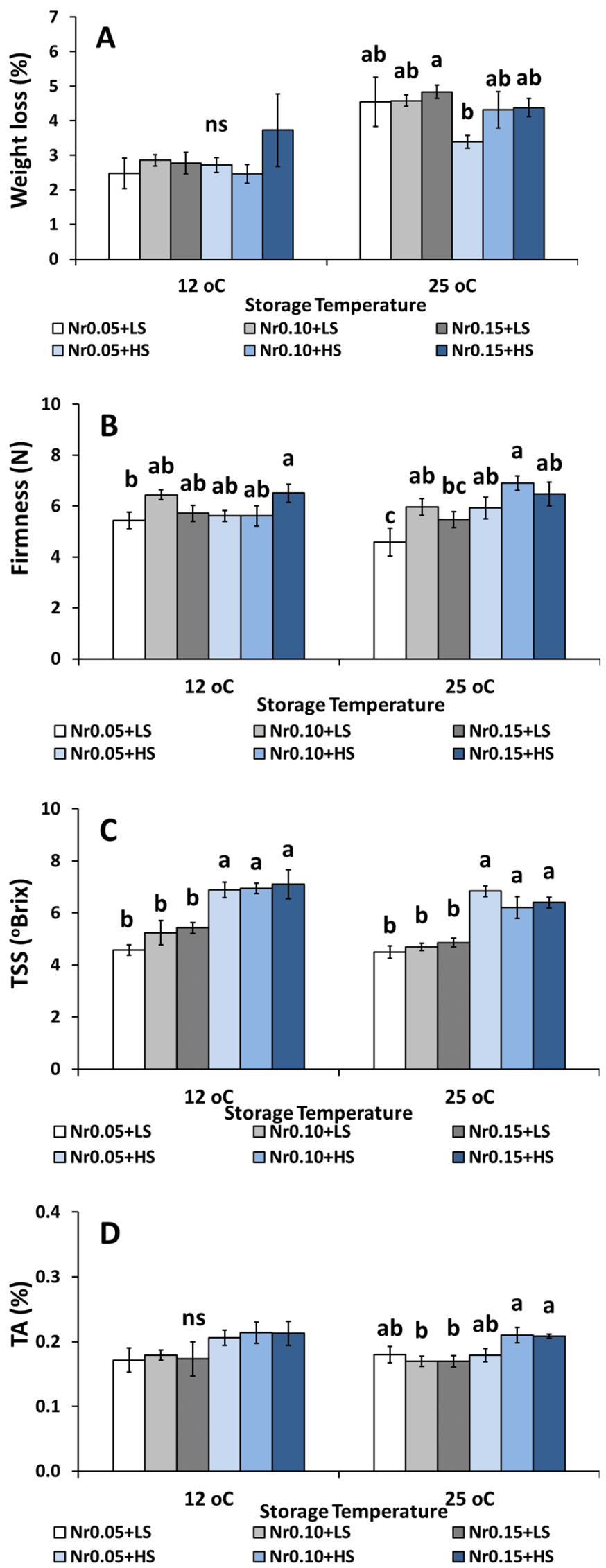

Figure 1. Cont. 


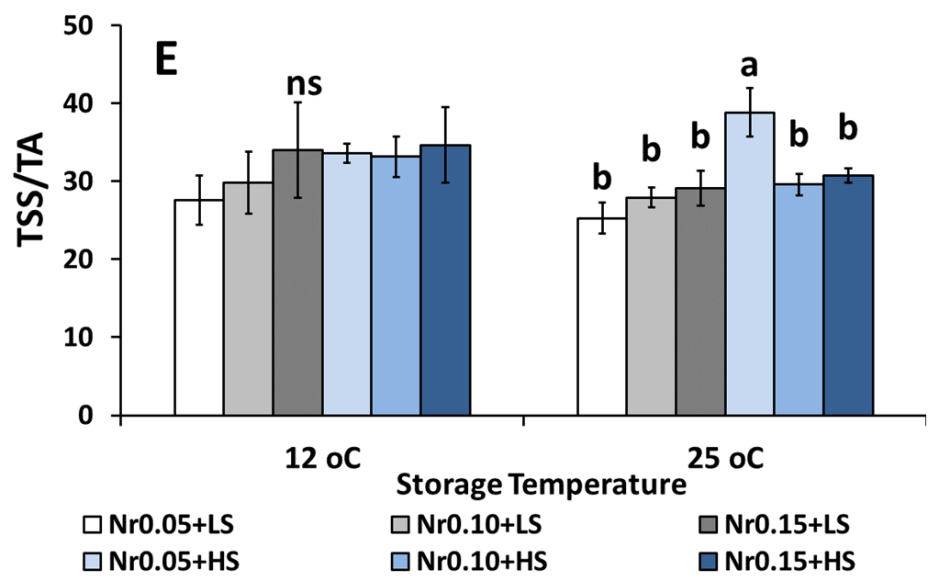

Figure 1. The effect of salinity (Sal) levels (LS: low salinity of $0 \mathrm{mM} \mathrm{NaCl}$; HS: high salinity of $50 \mathrm{mM}$ $\mathrm{NaCl})$ and the application of $\mathrm{NH}_{4} / \mathrm{N}_{\text {total }}$ ratios $(\mathrm{Nr} 0.05, \mathrm{Nr} 0.10$ and $\mathrm{Nr} 0.15)$ on tomato plants and the harvested fruit after storage for 7 days at $12{ }^{\circ} \mathrm{C}$ and $25^{\circ} \mathrm{C}$ for $(\mathbf{A})$ weight loss, (B) firmness, (C) total soluble solids-TSS, (D) titratable acidity-TA and (E) TSS/TA ratio. Non-significant differences are indicated by ns, while significant differences $(p<0.05)$ among treatments are indicated by different Latin letters according to Duncan's multiple range test. Error bars show SE $(n=8)$.
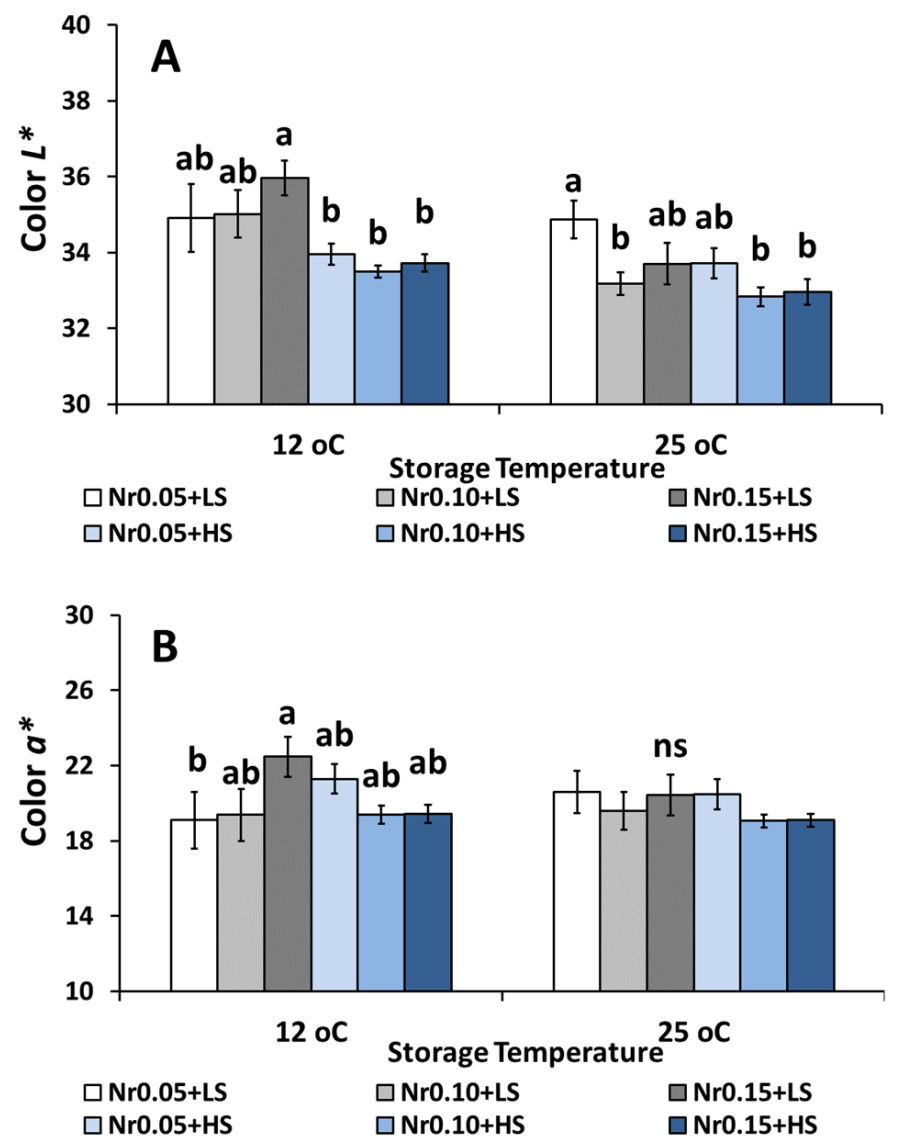

Figure 2. Cont. 

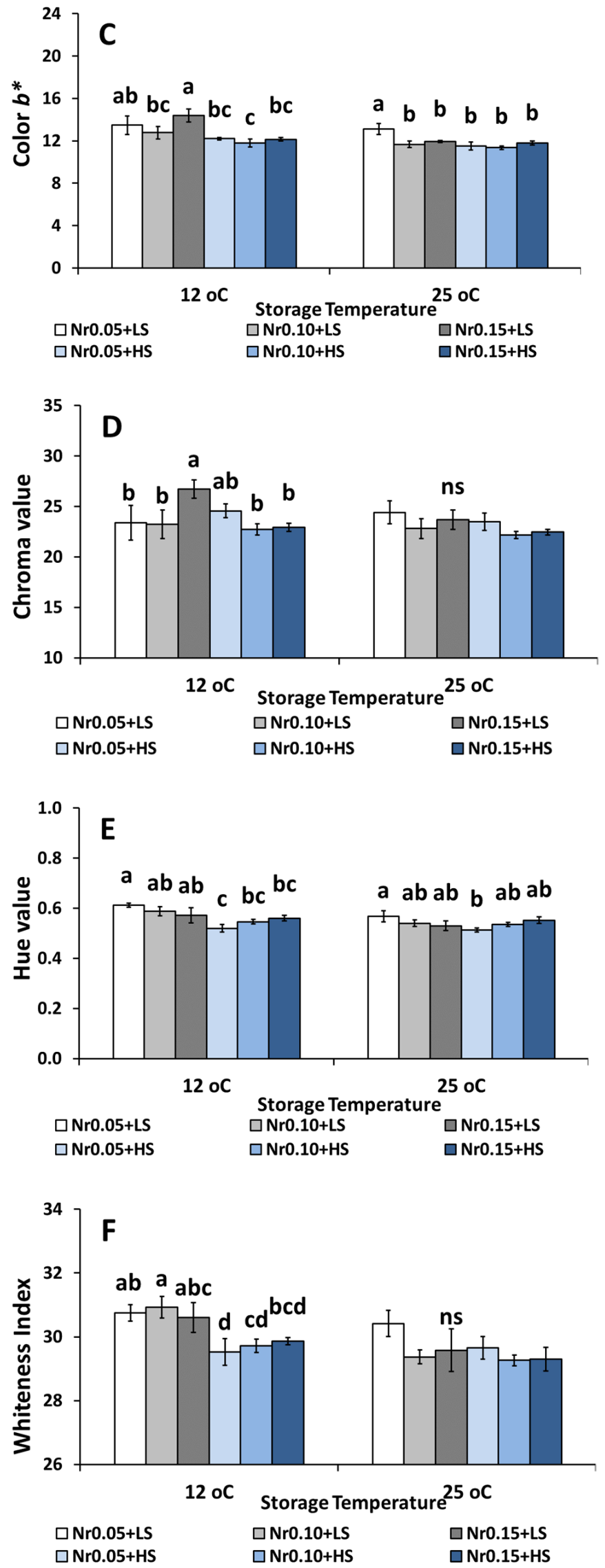

Figure 2. Cont. 


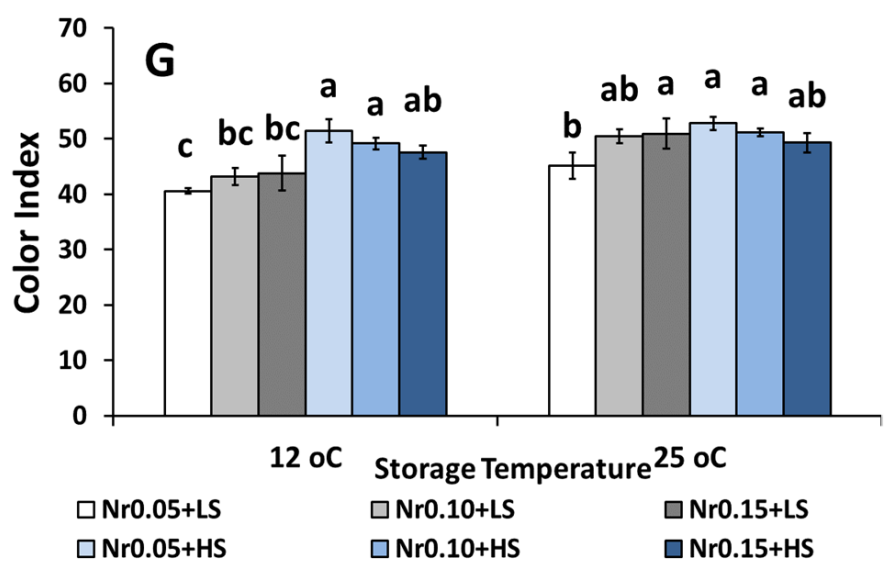

Figure 2. The effect of salinity (Sal) levels (LS: low salinity of $0 \mathrm{mM} \mathrm{NaCl} ; \mathrm{HS}$ : sigh salinity of $50 \mathrm{mM}$ $\mathrm{NaCl})$ and the application of $\mathrm{NH}_{4} / \mathrm{N}_{\text {total }}$ ratios $(\mathrm{Nr} 0.05, \mathrm{Nr} 0.10$, and $\mathrm{Nr} 0.15)$ on tomato plants and the harvested fruit after storage for 7 days at $12{ }^{\circ}$ and $25^{\circ} \mathrm{C}$ for fruit color parameters. (A) color $L^{*},(\mathbf{B})$ color $a^{*},(\mathbf{C})$ color $b^{*},(\mathbf{D})$ chroma, (E) Hue, (F) whiteness index, and (G) color index. Nonsignificant differences are indicated by $n s$, while significant differences $(p<0.05)$ among treatments are indicated by different Latin letters according to Duncan's multiple range test. Error bars show SE $(n=8)$.

At $12{ }^{\circ} \mathrm{C}$, fruit vitamin $\mathrm{C}$ was increased in the case of $\mathrm{Nr} 0.05+\mathrm{HS}$ (Figure 3A). Lycopene content was increased for $\mathrm{Nr} 0.10+$ HS plants in comparison to $\mathrm{Nr0.05}+\mathrm{LS}$, $\mathrm{Nr} 0.10+$ LS, Nr0.15 + LS and Nr0.15 + HS plants (Figure 3C). The content of $\beta$-carotene was increased in the cases of $\mathrm{Nr} 0.10+$ HS and $\mathrm{Nr} 0.15+$ HS in comparison to the $\mathrm{Nr} 0.05+\mathrm{LS}$ and Nr0.10 + LS plants (Figure 3D). The total phenols content in fruits was similar in both of the storage conditions in terms of temperature for both the saline and Nr treated plants (Figure 3B). At $25^{\circ} \mathrm{C}$, the content of $\beta$-carotene in fruits was increased in the saline-treated plants compared to the non-saline treated plants, independently of the Nr (Figure 3D). Similar to the total phenols content at $25^{\circ} \mathrm{C}$, the vitamin $\mathrm{C}$ and lycopene content remained unchanged for all treatments (Figure 3A,C).

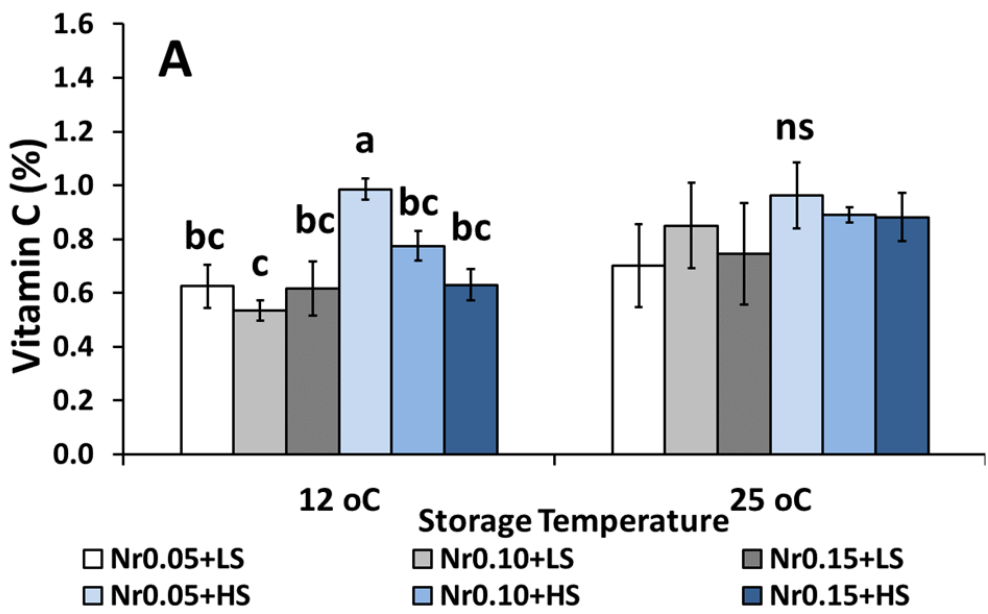

Figure 3. Cont. 

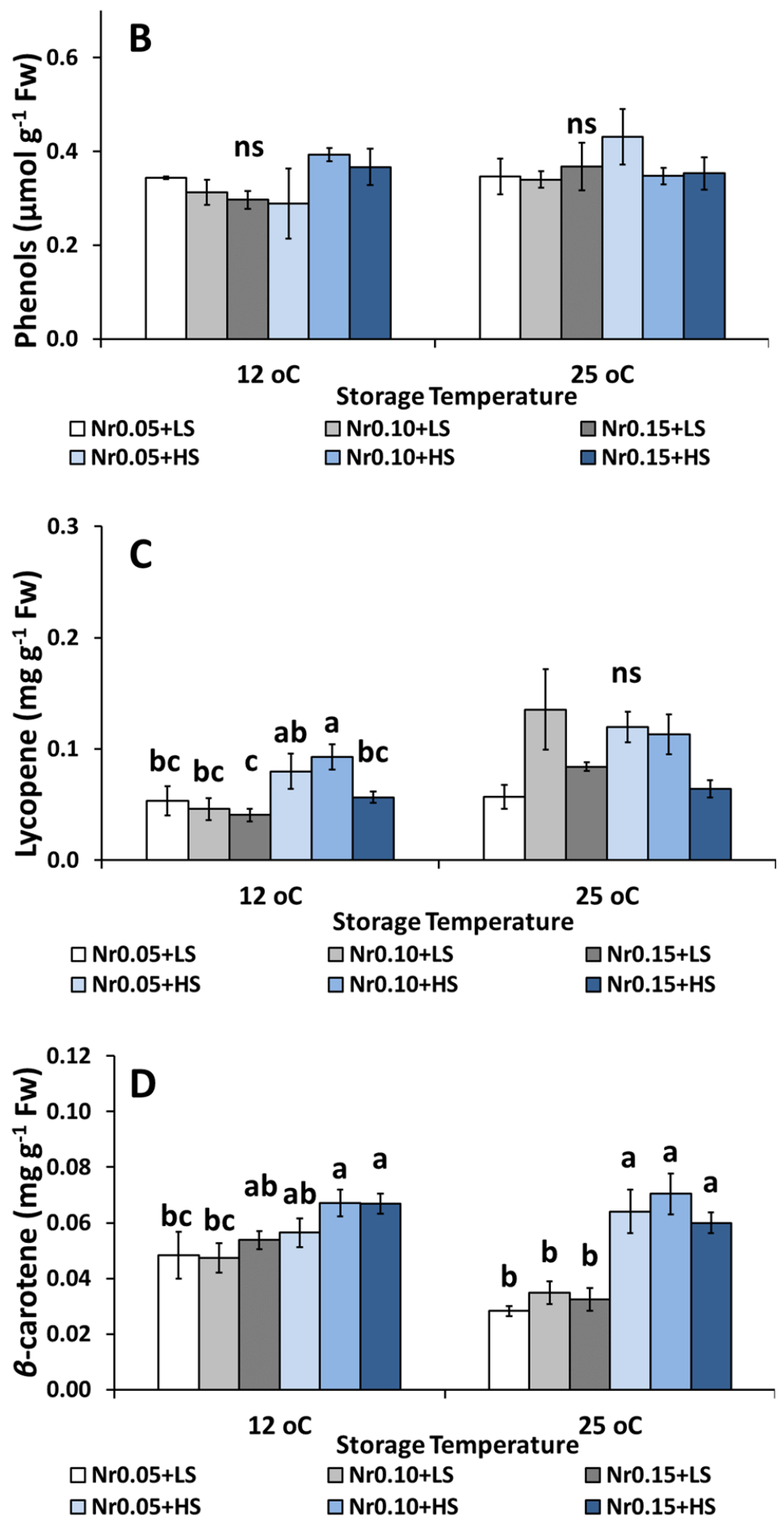

Figure 3. The effect of salinity (Sal) levels (LS: Low Salinity of $0 \mathrm{mM} \mathrm{NaCl}$; HS: High Salinity of $50 \mathrm{mM} \mathrm{NaCl})$ and the application of $\mathrm{NH}_{4} / \mathrm{N}_{\text {total }}$ ratios $(\mathrm{Nr} 0.05, \mathrm{Nr} 0.10$, and $\mathrm{Nr} 0.15)$ on tomato plants and the harvested fruit after storage for 7 days at $12{ }^{\circ} \mathrm{C}$ and $25^{\circ} \mathrm{C}$ for (A) vitamin $\mathrm{C},(\mathrm{B})$ total phenols, (C) lycopene and (D) $\beta$-carotene content. Non-significant differences are indicated by ns, while significant differences $(p<0.05)$ among treatments are indicated by different Latin letters according to Duncan's multiple range test. Error bars show SE $(n=8)$. 


\section{Discussion}

In the present study, which examined the impact of salinity and/or the ammoniumnitrate ratio (Nr0.05; $\mathrm{Nr} 0.10$ and $\mathrm{Nr} 0.15)$ on tomato cultivation, the results revealed that $\mathrm{NaCl}$-salinity significantly reduced the vegetative growth of tomato plants to the degree that the plant height, stem diameter, fruit fresh weight and total yield were directly affected. Other parameters such as the number of leaves per plant were not significantly influenced. The reduction in vegetative growth and yield under high salinity treatment is consistent with the results of Psaras et al. [40], Stamatakis et al. [41] and Costan et al. [35] concerning tomato crops. However, a different response was observed in an eggplant crop under salinity, where the vegetative parts and the leaf area were not significantly reduced by moderate levels of salinity and the most detrimental effects were observed on mean fruit weight and yield [42]. These findings further support the notion that the eggplant species is less sensitive to salinity stress than the tomato species. Furthermore, our findings showed that growing tomato plants in saline conditions increased the occurrence of BER symptoms by up to 9.4 percent, decreasing the marketability of the fruit significantly. Zhai et al. [43] found that high salinity increased the incidence of BER because high salinity is related to impaired $\mathrm{Ca}^{2+}$ uptake, which is the primary cause of BER symptoms.

The increased $\mathrm{NH}_{4}{ }^{+}$levels in the present study did not greatly affect the growth and yield of tomatoes, possible due to the low $\mathrm{NH}_{4}{ }^{+}$levels used. However, in the context of a higher $\mathrm{Nr}$ ratio in cucumber plants, plant yield was significantly decreased [20]. This decrease in yield due to the increased $\mathrm{Nr}$ within the $\mathrm{N}$ supply might be attributed to the inhibition of the uptake of $\mathrm{K}^{+}$and other cations [44]. In an early spring trial, providing $30 \%$ of total $\mathrm{N}$ in the form of $\mathrm{NH}_{4}{ }^{+}$significantly enhanced both the fresh and dry shoot weight of lettuce [19].

With respect to the nutrient solution, $\mathrm{pH}$ was both affected by the $\mathrm{Nr}$ ratio as well as the environmental conditions. A more intense drop in the $\mathrm{pH}$ value was observed under a high $\mathrm{Nr}$ and under the cold weather conditions of winter, while higher spring temperatures spring might alleviate the high $\mathrm{Nr}$ effect. In general, an increased $\mathrm{Nr}$ leads to $\mathrm{pH}$ reduction due to the release of $\mathrm{H}^{+}$ion into the nutrient/soil solution $[19,22]$. The impacts of a particular $\mathrm{NH}_{4}{ }^{+}-\mathrm{N} /$ total-N supply ratio may be quite different in an opened hydroponic system than in a closed one [19]. It is well known that too high or too low $\mathrm{pH}$ values in the root environment of plants may quickly result in nutrient deficiencies or toxicities, even when the supply of nutrients is optimal [32].

Nitrate accumulation either in the drainage solution or in the plants is related to low light density and low-cool temperatures, whereas these conditions were observed in the glasshouse from December to the middle of February. At the same time, the accumulation of nitrates in the drainage solution reached its peak during this period. The positive correlation of nitrate accumulation in the drainage solution and the plants with the weather conditions occurs mainly due to the incapability of the plants to absorb and/or metabolize the nitrates due to reduced photosynthesis rates and the reduced activity of the enzyme nitrate reductase under these conditions (poor light density, cool temperatures) [45]. Furthermore, ammonium increases the acidity of the cells and decreases the permeability of the cell membrane, leading to an inhibition of nitrate absorption and reduction. This is consistent with the present study, where the highest accumulation of nitrate in the drainage solution was observed under high ammonium rates.

In terms of leaf nutrient content, the results showed that the levels of $\mathrm{Ca}$ in the leaves did not change regardless of the treatments used. This is consistent with the findings of Tuna et al., who claimed that $\mathrm{Ca}$ is replaced by $\mathrm{Na}$ in cell membranes during salt stress [46], and previous studies on tomato crops subjected to salinity [35]. As in the case of $\mathrm{Ca}, p$ and $\mathrm{Cu}$ levels also remained unaffected by the tested treatments. Several previous studies have shown that salinity either increases [35] or has no effect on $p$ uptake [47,48]. Additionally, it has been reported that $p$ levels increased in a mountainous population of Cichorium spinosum but were unaffected in the seaside population when plants were subjected to the application of increased $\mathrm{Nr}$ [22]. As a result, it is reasonable to believe that a variety of factors influence 
$p$ uptake, including temperature, water availability in the root environment, species and even cultivar [49]. Salinity decreased $\mathrm{K}$ levels in the present study, and these data indicate that $\mathrm{K}^{+}$and $\mathrm{Na}^{+}$are in competition. The decrease in $\mathrm{K}$ levels in leaf tissues is consistent with the findings of del Amor et al. and Atluntas et al., who revealed that a decrease in $\mathrm{K}$ content is balanced out by an increase in Na content [50,51]. Similarly, when plants were cultivated in a saline environment, $\mathrm{Na}^{+}$levels are much greater, which is confirmed by the results of del Amor et al. [50] and Costan et al. [35]. The Zn levels in the present study were increased with the application of $50 \mathrm{mM} \mathrm{NaCl}$ and with high $\mathrm{Nr}$ levels, in comparison to saline with low $\mathrm{Nr}$ treatments and/or non-saline conditions, indicating the interactive role between ammonium and sodium ions. Indeed, salinity itself had previously been shown to increase Zn levels in tomato leaves [35] and lavender leaves [52]. Finally, the fact that $\mathrm{Fe}$ and $\mathrm{Mn}$ content increased with the application of saline and high $\mathrm{Nr}$ is in agreement with the findings of Turan et al., who have stated that salinity increases the concentration of $\mathrm{Mn}$ in the shoot and root tissues of maize [53]. It has also been reported that increased $\mathrm{Nr}$ levels result in decreased $\mathrm{Zn}, \mathrm{Cu}$ and $\mathrm{Mn}$ levels in C. spinosum [22].

Regarding fruit nutrient status, Ca content was negatively influenced with an increasing $\mathrm{Nr}$ due to $\mathrm{NH}_{4}{ }^{+} / \mathrm{Ca}^{2+}$ competition, while $\mathrm{Na}^{+} / \mathrm{K}^{+}$and $\mathrm{Na}^{+} / \mathrm{Ca}^{2+}$ competition also occurred under high salinity. Reduced Ca content in fruits can lead to BER disorder, which is quite common in the case of tomato crops. In the present study, this disorder appeared above the third cluster, and ranged from $7-9.4 \%$ in the fruits where salinity was applied, with $\mathrm{Nr} 0.05$ giving the lowest value, while the percentage of the fruits with BER was increased at higher Nr ratios. Potassium content decreased with salinity, especially at increased $\mathrm{Nr}$ ratios, with the application of $\mathrm{Nr} 0.15$ under high salinity giving the lowest value. This indicates the strong competition of $\mathrm{K}^{+}$with both $\mathrm{NH}_{4}{ }^{+}$and $\mathrm{Na}^{+}$in this case. Similar results were found by Adler and Wilcox in a study with respect to salt-stressed muskmelon plants, suggesting that the influx of $\mathrm{Na}$ is regulated by $\mathrm{K} / \mathrm{Na}$ selectivity and exchanges across cell membranes [54]. Additionally, an increased $\mathrm{Nr}$ ration resulted in the decreased content of several minerals in cucumber fruits [44]. Phosphorus content was reduced by salinity at low Nr. In general, salinity decreases the concentration of $p$ in plant tissue [35,55], with several contradictions being present in previous studies, as it has been indicated that salinity either increased or has no effect on $p$ uptake. Plant growing conditions, the plant type and even the cultivar play an important role in $p$ accumulation [49]. In the present study, the reduced Mg content in the context of high salinity at $\mathrm{Nr} 0.05$ indicates competition between $\mathrm{Mg}$ and $\mathrm{Na}$ but not with ammonium ions. The nitrogen content of the fruits decreased with salinity at low and moderate $\mathrm{Nr}$ levels, which could be attributed to the reduced nitrate uptake and translocation into the plant under high salinity conditions. In the present study, the nitrogen content of the fruits was restored when nitrogen was supplied in ammonium form ( $\mathrm{Nr} 0.15)$. The application of an increased $\mathrm{Nr}$ ratio to hydroponically grown cucumber resulted in decreased $\mathrm{Ca}, \mathrm{Mg}, \mathrm{K} \mathrm{Cu}, \mathrm{Mn}$ and $\mathrm{NO}_{3}$ content in cucumber fruits [44].

Low $\mathrm{pH}$ values are considered to be responsible for increased $\mathrm{Al}$ released from the perlite into the drainage solution; this is consistent with the results of the present study, which indicated the $\mathrm{Al}$ increment at low $\mathrm{pH}$ values (increased $\mathrm{Nr}$ ). Iron content was increased by increasing the $\mathrm{Nr}$ under low salinity. This is consistent with Flores et al. [56], who, in the context of a tomato crop, indicated an increased correlation between nitrogen assimilation and the levels of Fe by adding nitrogen in ammonium form, resulting in reduced energy requirements for plant growth. Salt stress reduced the $\mathrm{Cu}$ content, and this has also been evidenced in salt-stressed maize grown in both soil [57] and solution cultures [58]. Manganese content was suppressed by salinity and a high $\mathrm{Nr}$ ratio ( $\mathrm{Nr} 0.10$; $\mathrm{Nr} 0.15)$. Salinity reduces Mn content in the shoot tissue of horticultural crops, for example, squash [59] and corn [57], according to most research, regardless of whether they were grown in soils or in solution cultures. However, some studies on tomato crops have shown that salinity either has no effect [60] or increases the Mn content [59] of leaves or shoot tissue. 
Several quality characteristics of the tomato are influenced by salinity and the $\mathrm{Nr}$ ratio. Citric acid content in general significantly increased with salinity. The increased acidity of salt-stressed vegetable crops has been reported for tomatoes [35,41] and eggplants [42] and has been associated with an increase in the level of organic acids which are involved in the osmoregulation mechanism of plants under high salinity conditions [61]. The total soluble solid contents were also significantly higher under high salinity, and were not affected by either the Nr or by the storage temperatures. The beneficial effects of salinity on soluble solids content are consistent with prior research [40,62], though it should be noted that when results are expressed on a fresh weight basis, such an increase could be due to a concentration effect rather than an increase in total soluble solids [63]. Additionally, Ben and Kafkafi [64] previously reported that TSS in melon fruit remained the same when the melon plants exposed to different Nr levels. Fruit firmness was influenced by both salinity and the Nr ratio. More specifically, salinity had a positive effect on fruit firmness at the point of harvest, while the $\mathrm{Nr}$ ratio had a positive effect only under high salinity conditions or following storage. As expected, the dehydration due to weight loss of the tomatoes stored at room temperature was much more intense than the ones that were stored at $12{ }^{\circ} \mathrm{C}$ and remained unaffected by salinity or $\mathrm{Nr}$.

The vitamin C content of the fruits was higher due to salinity at the point of harvest, while an increased $\mathrm{Nr}$ ratio decreased the vitamin $\mathrm{C}$ content under high salinity conditions following storage for a week at $12{ }^{\circ} \mathrm{C}$. Storage at room temperature diminished the effect of both salinity and $\mathrm{Nr}$ on vitamin $\mathrm{C}$ content. These enhancing effects of salinity on fruit firmness and vitamin $C$ content of tomato are in agreement with the results of other investigators $[65,66]$. The effect on concentration impacts originates from reduced water content in the fruit due to plant adaptation to salinity. Other investigators [32] obtained fruits with lower firmness (softer fruits) when the $\mathrm{Nr}$ was increased by more than 0.15 .

Plant stress factors such as salinity play an important role in terms of the antioxidant content of fruits. The total phenol content was increased by salinity at low $\mathrm{Nr}$ at the point of harvest. Moreover, the content of lycopene and $\beta$-carotene increased in fruits derived from the saline treated plants following storage at $12{ }^{\circ} \mathrm{C}$. However, the $\mathrm{Nr}$ levels seemed not to affect the fruits' antioxidant-related measures (vitamin $\mathrm{C}$, lycopene, $\beta$-carotene and total phenolics) at the harvesting stage, but decreased vitamin $C$ and lycopene levels were found in fruits stored at $12{ }^{\circ} \mathrm{C}$ for 1 week in relation to high salinity and $\mathrm{Nr}$ ratio treatments. Tomato fruit has been found to contain more total phenols when grown in saline conditions [62], while lavender plants have been found to contain more total phenols when grown in salinity levels of more than $50 \mathrm{mM} \mathrm{NaCl}$ [52]. Similar to the findings of our study, Serio et al. found that lycopene content was unchanged in tomato fruit when plants were exposed to salinity [67]; however, Moya et al. found the opposite [62]. These contradictory outcomes could be related to the severity of salinity stress, since different $\mathrm{NaCl}$ levels were applied in these studies and mild stress $\left(4.5 \mathrm{dS} \mathrm{m}^{-1}\right)$ appears to be favorable to tomato fruit lycopene content when compared to more severe stress $\left(6.0 \mathrm{dS} \mathrm{m}^{-1}\right)$.

Moderate salinity enhances fruit carotenoid content in tomato [66], possibly via the activation of the biosynthetic pathway, inducing up-regulation of the genes encoding the enzymes related to lycopene levels [68]. Concerning the tomatoes that were stored at room temperature, only $\beta$-carotene content was increased due to the increased EC of the nutrient solution. Similar results regarding salinity and increased antioxidant activity were observed in pepper fruit but only in mature red fruits while at the green (immature) stage, the results were controversial [69]. Furthermore, in previous studies, the antioxidant status of tomatoes increased after ten days of storage in similar conditions $\left(15^{\circ} \mathrm{C}\right.$ and $25{ }^{\circ} \mathrm{C}$ respectively), whereas the lycopene content was 1.8-fold higher than that of refrigerated tomatoes $\left(7^{\circ} \mathrm{C}\right)$. This could be explained due to a reduction in the water content of the fruits stored as well as the slowdown of the fruits' metabolism in chilled temperatures [70].

Regarding the color of tomatoes, salinity decreased fruit lightness at the point of harvest, as reported in previous studies on tomato [66], while the values $a^{*}$ (red colour) and $b^{*}$ (yellow colour) were not affected. After storage at $12{ }^{\circ} \mathrm{C}$ for a week, all color factors were 
improved with increasing $\mathrm{Nr}$, while salinity in general had a negative effect. Following storage at $25^{\circ} \mathrm{C}$, salinity decreased the lightness of the tomatoes at high and low $\mathrm{Nr}$. This is not consistent with research in which salinity in general improved color factors due to the increase of tomato pigments (lycopene and $\beta$-carotene) [30], although, in our study, only $\beta$-carotene increased at high salinity levels and lycopene was affected to a lesser extent, with this being in agreement with previous reports on tomatoes under saline conditions [35].

Following organoleptic tests, the increased $\mathrm{Nr}$ improved the fruits' appearance, color, marketability and aroma, with significant higher scores associated with fruit color according to the panelists, and with the fruits cultivated under $\mathrm{Nr} 0.10$ and $\mathrm{Nr} 0.15$ getting more positive records than the $\mathrm{Nr} 0.05$ sample. The fruit of the $50 \mathrm{mM} \mathrm{NaCl}$ treated plants performed better in terms of aroma and sweetness scores. In addition, the texture, satisfaction level and marketability of the salinity treated fruit received the best numerical score. The results of the questionnaires back up previously stated findings that salinity has a positive effect on some quality measures of tomato fruit. According to research, increased salt levels in NS result in increased fruit dry matter, sugars and organic acid content, as well as improving flavor and maintaining firmness in hydroponically produced tomato plants [71,72]. The findings of our study highlight the relevance of organoleptic tests in addition to chemical composition investigations because consumer approval is critical for fruit marketability.

The storage temperature did not greatly affect fruit metabolism due to a short duration of 7 days and the ripening stage of the fruits (red fruits). Indeed, fruits stored at chilled conditions at $12{ }^{\circ} \mathrm{C}$, in comparison to those strored at $25^{\circ} \mathrm{C}$, revealed lower fruit weight loss, as chilled conditions delayed the plant's metabolism and the evapotranspiration of the plant tissue. Neither storage at $12{ }^{\circ} \mathrm{C}$ or $25^{\circ} \mathrm{C}$, nor $\mathrm{Nr}$ levels affected the total soluble solids content of the fruits, while salinity increased TSS. The vitamin $\mathrm{C}$ content at $12{ }^{\circ} \mathrm{C}$ was increased at low $\mathrm{Nr}$ levels with the application of salinity, which is in agreement with previous studies which reported an increase in vitamin $C$ content in tomato fruit grown hydroponically under saline conditions [41]. However, Vitamin $C$ was unaffected in the case of fruits stored at $25^{\circ} \mathrm{C}$. Storage for $7 \mathrm{~d}$ showed that the content of vitamin $\mathrm{C}$, lycopene, $\beta$-carotene and total phenols was almost the same or slightly higher than prior to storage, regardless of storage temperature, $\mathrm{Nr}$ level and salinity level (with exception of lycopene and $\beta$-carotene content, which were increased with the application of salinity at $25^{\circ} \mathrm{C}$ ), a finding which is very important for the preservation of fruit quality. Similar to the present results, it has been reported in previous studies that lycopene content in tomatoes stored at $15^{\circ} \mathrm{C}$ and $25^{\circ} \mathrm{C}$ was higher (1.8-fold) than in refrigerated tomatoes $\left(7^{\circ} \mathrm{C}\right)$ [70]. Fruit quality is influenced by genotype. Manzo et al. found substantial differences in post storage quality between short and long shelf-life cultivars [73], while Petric et al. emphasized the need of maintaining good post-storage quality at the right temperature [74]. Following storage at $25^{\circ} \mathrm{C}$, no differences were observed between the salinity levels concerning color attributes $\left(L^{*}, a^{*}, b^{*}\right)$, owing to the fact that all the tested fruit were harvested at the mature red stage and that no further color development should be expected after this stage, but also the fact that lycopene content, which is responsible for the red color of the fruits, remained unaffected for all the tested treatments [73].

\section{Conclusions}

This study explored the effects of salinity on hydroponically grown tomato plants and evaluated whether the application of different $\mathrm{Nr}$ levels affected the negative effects posed by $\mathrm{NaCl}$ stress. Moreover, the postharvest performance of fruit stored either in chilled conditions or at shelf life was examined. Despite the negative effects of salinity on plant growth as well as yield, the fruit fresh weight was only decreased significantly with the application of high Nr. Salinity and high ammonium ratios (high Nr) induced nutrient imbalances in the fruit, such as $\mathrm{Ca}$, and affected BER in fruits, the symptoms of which appeared on the fruits above the third cluster after five months of tomato cultivation (March) and did not affect the marketability of the examined fruits. It was profound that 
salinity improved and/or maintained various fruit quality related attributes, including total acid content, total soluble solids content, firmness and vitamin C content, while an increased $\mathrm{Nr}$ also preserved fruit firmness after storage. These characteristics are considered favorable by consumers and are important in terms of fruit storability and postharvest life. Lycopene, $\beta$-carotene and vitamin $C$ were increased by salinity during harvesting or following storage. The organoleptic control showed that tomato fruits cultivated in an increasing $\mathrm{Nr}$ or under high salinity were more preferable to consumers due to the improvement of some organoleptic characteristics. Salinity enhances fruit quality and improves the organoleptic characteristics of the crop, while an appropriate Nr ratio may restrict the negative effects of salinity on the nutritional status of plants by regulating the $\mathrm{pH}$ in hydroponic systems. More research on this topic in needed in order to look into the interactions between different $\mathrm{Nr}$ ratios and $\mathrm{NaCl}$ salinity in other fruits and vegetables, and also to determine whether the application of minerals and which application methods are effective tools to reduce the harmful impacts of salt.

Supplementary Materials: The following supporting information can be downloaded at: https: / / www.mdpi.com/article/10.3390/agronomy12020386/s1, Figure S1. Average, minimum and maximum air relative humidity $(\mathrm{RH})$ and temperature $\left({ }^{\circ} \mathrm{C}\right)$ during the experiment. Figure $\mathrm{S} 2$. The effect of salinity (Sal) levels (LS: Low Salinity of $0 \mathrm{mM} \mathrm{NaCl}$; HS: High Salinity of $50 \mathrm{mM} \mathrm{NaCl}$ ) and the application of $\mathrm{NH}_{4} / \mathrm{N}_{\text {total }}$ ratios $(\mathrm{Nr} 0.05, \mathrm{Nr} 0.10$, and $\mathrm{Nr} 0.15)$ on the fluctuation of the electrical conductivity $\left(\mathrm{EC} ; \mathrm{mS} \mathrm{cm}^{-1}\right), \mathrm{pH}$ and nitrates content of the drainage solution during cultivation.

Author Contributions: Conceptualization, N.T. and A.S.; methodology, N.T. and A.S.; software, A.C. and A.S.; validation, N.T., A.C. and A.S.; formal analysis, A.C., A.S. and N.T.; investigation, G.P., A.C., A.S. and N.T.; resources, N.T. and A.S.; data curation, A.C. and A.S.; writing-original draft preparation, A.C. and N.T.; writing-review and editing, A.C., A.S. and N.T.; visualization, A.C. and A.S.; supervision, N.T.; project administration, N.T.; funding acquisition, N.T. and A.S. All authors have read and agreed to the published version of the manuscript.

Funding: This research received no external funding.

Institutional Review Board Statement: Not applicable.

Data Availability Statement: Not applicable.

Conflicts of Interest: The authors declare no conflict of interest.

\section{References}

1. Wu, G.; Zhang, C.; Chu, L.Y.; Shao, H.B. Responses of higher plants to abiotic stresses and agricultural sustainable development. J. Plant Interact. 2007, 2, 135-147. [CrossRef]

2. Munns, R.; Tester, M. Mechanisms of salinity tolerance. Annu. Rev. Plant Biol. 2008, 59, 651-681. [CrossRef] [PubMed]

3. Shahbaz, M.; Ashraf, M. Improving Salinity Tolerance in Cereals. Crit. Rev. Plant Sci. 2013, 32, 237-249. [CrossRef]

4. Shrivastava, P.; Kumar, R. Soil salinity: A serious environmental issue and plant growth promoting bacteria as one of the tools for its alleviation. Saudi J. Biol. Sci. 2015, 22, 123-131. [CrossRef] [PubMed]

5. Yildirim, E.; Taylor, A.G. Effect of biological treatments on growth of bean plants under salt stress. Annu. Rep. Bean Improv. Coop. 2005, 48, 176-177.

6. Hendawy, S.F.; Khalid, K.A. Response of sage (Salvia officinalis L.) plants to zinc application under different salinity levels. J. Appl. Sci. Res. 2005, 1, 147-155.

7. Gama, P.B.S.S.; Inanaga, S.; Tanaka, K.; Nakazawa, R. Nakazawa Physiological response of common bean (Phaseolus vulgaris L.) seedlings to salinity stress. Afr. J. Biotechnol. 2007, 6, 079-088.

8. Jamil, M.; Rehman, S.; Rha, E.S. Salinity effect on plant growth, PSII photochemistry and chlorophyll content in sugar beet (Beta vulgaris L.) and cabbage (Brassica oleracea capitata L.). Pak. J. Bot. 2007, 39, 753-760.

9. Memon, S.A.; Hou, X.; Wang, L.J. Morphlogical analysis of salt stress response of pak choi. Electron. J. Environ. Agric. Food Chem. 2010, 9, 248-254.

10. Pinheiro, D.T.; Delazari, F.; Nick, C.; Mattiello, E.M.; dos Santos Dias, D.C.F. Emergence and vegetative development of melon in function of the soil salinity. Aust. J. Crop. Sci. 2019, 13, 458-464. [CrossRef]

11. Petretto, G.L.; Urgeghe, P.P.; Massa, D.; Melito, S. Effect of salinity (NaCl)on plant growth, nutrient content, and glucosinolate hydrolysis products trends in rocket genotypes. Plant Physiol. Biochem. 2019, 141, 30-39. [CrossRef] [PubMed] 
12. Belmecheri-Cherifi, H.; Albacete, A.; Martínez-Andújar, C.; Pérez-Alfocea, F.; Abrous-Belbachir, O. The growth impairment of salinized fenugreek (Trigonella foenum-graecum L.) plants is associated to changes in the hormonal balance. J. Plant Physiol. 2019, 232, 311-319. [CrossRef] [PubMed]

13. Ramos-Sotelo, H.; Valdez-Ortiz, Á.; Germán-Báez, L.J.; Fierro-Sañudo, J.F.; León-Cañedo, J.A.; Alarcón-Silvas, S.G.; Reyes-Moreno, C.; Páez-Osuna, F. Quality of lettuce Lactuca sativa (var. Tropicana M1) grown with two low-salinity shrimp effluents. Food Chem. X 2019, 2, 100027. [CrossRef] [PubMed]

14. Sarabi, B.; Fresneau, C.; Ghaderi, N.; Bolandnazar, S.; Streb, P.; Badeck, F.W.; Citerne, S.; Tangama, M.; David, A.; Ghashghaie, J. Stomatal and non-stomatal limitations are responsible in down-regulation of photosynthesis in melon plants grown under the saline condition: Application of carbon isotope discrimination as a reliable proxy. Plant Physiol. Biochem. 2019, 141, 1-19. [CrossRef]

15. Kapoor, K.; Srivastava, A. Assessment of salinity tolerance of Vigna mungo var. Pu-19 using ex vitro and in vitro methods. Asian J. Biotechnol. 2010, 2, 73-85. [CrossRef]

16. Piñero, M.C.; Pérez-Jiménez, M.; López-Marín, J.; del Amor, F.M. Fruit quality of sweet pepper as affected by foliar Ca applications to mitigate the supply of saline water under a climate change scenario. J. Sci. Food Agric. 2018, 98, 1071-1078. [CrossRef]

17. Chrysargyris, A.; Papakyriakou, E.; Petropoulos, S.A.; Tzortzakis, N. The combined and single effect of salinity and copper stress on growth and quality of Mentha spicata plants. J. Hazard. Mater. 2019, 368, 584-593. [CrossRef]

18. Incerti, A.; Navari-Izzo, F.; Pardossi, A.; Izzo, R. Seasonal variations in polyphenols and lipoic acid in fruits of tomato irrigated with sea water. J. Sci. Food Agric. 2009, 89, 1326-1331. [CrossRef]

19. Savvas, D.; Passam, H.C.; Olympios, C.; Nasi, E.; Moustaka, E.; Mantzos, N.; Barouchas, P. Effects of ammonium nitrogen on lettuce grown on pumice in a closed hydroponic system. HortScience 2006, 41, 1667-1673. [CrossRef]

20. Kotsiras, A.; Olympios, C.M.; Passam, H.C. Effects of nitrogen form and concentration on yield and quality of cucumbers grown on rockwool during spring and winter in southern Greece. J. Plant Nutr. 2005, 28, 2027-2035. [CrossRef]

21. Santamaria, P.; Elia, A. Producing nitrate-free endive heads: Effect of nitrogen form on growth, yield, and ion composition of endive. J. Am. Soc. Hortic. Sci. 1997, 122, 140-145. [CrossRef]

22. Chatzigianni, M.; Alkhaled, B.; Livieratos, I.; Stamatakis, A.; Ntatsi, G.; Savvas, D. Impact of nitrogen source and supply level on growth, yield and nutritional value of two contrasting ecotypes of Cichorium spinosum L. grown hydroponically. J. Sci. Food Agric. 2018, 98, 1615-1624. [CrossRef] [PubMed]

23. Perner, H.; Schwarz, D.; Krumbein, A.; George, E. Influence of sulfur supply, ammonium nitrate ratio, and arbuscular mycorrhizal colonization on growth and composition of Chinese chive. Sci. Hortic. 2011, 130, 485-490. [CrossRef]

24. Fallovo, C.; Rouphael, Y.; Rea, E.; Battistelli, A.; Colla, G. Nutrient solution concentration and growing season affect yield and quality of Lactuca sativa L. var. acephala in floating raft culture. J. Sci. Food Agric. 2009, 89, 1682-1689. [CrossRef]

25. Rouphael, Y.; Kyriacou, M.C.; Petropoulos, S.A.; De Pascale, S.; Colla, G. Improving vegetable quality in controlled environments. Sci. Hortic. 2018, 234, 275-289. [CrossRef]

26. Palaniswamy, U.R.; Bible, B.B.; McAvoy, R.J. Oxalic acid concentrations in Purslane (Portulaca oleraceae L.) is altered by the stage of harvest and the nitrate to ammonium ratios in hydroponics. Sci. Hortic. 2004, 102, 267-275. [CrossRef]

27. Boschiero, B.N.; Mariano, E.; Azevedo, R.A.; Ocheuze Trivelin, P.C. Influence of nitrate-ammonium ratio on the growth, nutrition, and metabolism of sugarcane. Plant Physiol. Biochem. 2019, 139, 246-255. [CrossRef]

28. Szczerba, M.W.; Britto, D.T.; Balkos, K.D.; Kronzucker, H.J. Alleviation of rapid, futile ammonium cycling at the plasma membrane by potassium reveals $\mathrm{K}^{+}$-sensitive and -insensitive components of $\mathrm{NH}_{4}^{+}$transport. J. Exp. Bot. 2008, 59, 303-313. [CrossRef]

29. Maggio, A.; Raimondi, G.; Martino, A.; De Pascale, S. Salt stress response in tomato beyond the salinity tolerance threshold. Environ. Exp. Bot. 2007, 59, 276-282. [CrossRef]

30. Giannakoula, A.E.; Ilias, I.F. The effect of water stress and salinity on growth and physiology of tomato (Lycopersicon esculentum Mill.). Arch. Biol. Sci. 2013, 65, 611-620. [CrossRef]

31. Claussen, W. Growth, water use efficiency, and proline content of hydroponically grown tomato plants as affected by nitrogen source and nutrient concentration. Plant Soil 2002, 247, 199-209. [CrossRef]

32. Akl, I.A.; Savvas, D.; Papadantonakis, N.; Lydakis-Simantiris, N.; Kefalas, P. Influence of Ammonium to Total Nitrogen Supply Ratio on Growth, Yield and Fruit Quality of Tomato Grown in a Closed Hydroponic System. Eur. J. Hortic. Sci. 2003, 68, 204-211.

33. Chrysargyris, A.; Antoniou, O.; Athinodorou, F.; Vassiliou, R.; Papadaki, A.; Tzortzakis, N. Deployment of olive-stone waste as a substitute growing medium component for Brassica seedling production in nurseries. Environ. Sci. Pollut. Res. 2019, 26, 35461-35472. [CrossRef]

34. Eaton, A.; Clesceri, L.; Rice, E.; Greenberg, A. Standard Methods for Examination of Water and Wastewater: Centennial Edition, 1368; American Public Health Association: Washington, DC, USA, 2005.

35. Costan, A.; Stamatakis, A.; Chrysargyris, A.; Petropoulos, S.A.; Tzortzakis, N. Interactive effects of salinity and silicon application on Solanum lycopersicum growth, physiology and shelf life of fruit produced hydroponically. J. Sci. Food Agric. 2019, 100, 732-743. [CrossRef] [PubMed]

36. Xylia, P.; Clark, A.; Chrysargyris, A.; Romanazzi, G.; Tzortzakis, N. Quality and safety attributes on shredded carrots by using Origanum majorana and ascorbic acid. Postharvest Biol. Technol. 2019, 155, 120-129. [CrossRef]

37. Chrysargyris, A.; Rousos, C.; Xylia, P.; Tzortzakis, N. Vapour application of sage essential oil maintain tomato fruit quality in breaker and red ripening stages. Plants 2021, 10, 2645. [CrossRef] [PubMed] 
38. AOAC. Official Methods of Analysis of AOAC International; Horwitz, W., Latimer, G., Eds.; AOAC International: Gaithersburg, MD, USA, 2016.

39. Nagata, M.; Yamashita, I. Simple Method for Simultaneous Determination of Chlorophyll and Carotenoids in Tomato Fruit. Nippon. Shokuhin Kogyo Gakkaishi 1992, 39, 925-928. [CrossRef]

40. Psarras, G.; Bertaki, M.; Chartzoulakis, K. Response of greenhouse tomato to salt stress and $\mathrm{K}^{+}$supplement. Plant Biosyst. 2008, 142, 149-153. [CrossRef]

41. Stamatakis, A.; Papadantonakis, N.; Savvas, D.; Lydakis-Simantiris, N.; Kefalas, P.; Savvas, D.; Lydakis-Simantiris, N.; Kefalas, P. Effects of silicon and salinity on fruit yield and quality of tomato grown hydroponically. Acta Hortic. 2003, 609, 141-147. [CrossRef]

42. Savvas, D.; Lenz, F. Response of eggplants grown in recirculating nutrient solution to salinity imposed prior to the start of harvesting. J. Hortic. Sci. Biotechnol. 2000, 75, 262-267. [CrossRef]

43. Zhai, Y.; Yang, Q.; Hou, M. The effects of saline water drip irrigation on tomato yield, quality, and blossom-end rot incidence-A 3a case study in the South of China. PLoS ONE 2015, 10, e0142204.

44. Kotsiras, A.; Olympios, C.M.; Drosopoulos, J.; Passam, H.C. Effects of nitrogen form and concentration on the distribution of ions within cucumber fruits. Sci. Hortic. 2002, 95, 175-183. [CrossRef]

45. Balotf, S.; Kavoosi, G. Differential nitrate accumulation, nitrate reduction, nitrate reductase activity, protein production and carbohydrate biosynthesis in response to potassium and sodium nitrate. Afr. J. Biotechnol. 2011, 10, 17973-17980.

46. Tuna, A.L.; Kaya, C.; Ashraf, M.; Altunlu, H.; Yokas, I.; Yagmur, B. The effects of calcium sulphate on growth, membrane stability and nutrient uptake of tomato plants grown under salt stress. Environ. Exp. Bot. 2007, 59, 173-178. [CrossRef]

47. Kostic, L.; Nikolic, N.; Bosnic, D.; Samardzic, J.; Nikolic, M. Silicon increases phosphorus (P) uptake by wheat under low P acid soil conditions. Plant Soil 2017, 419, 447-455. [CrossRef]

48. Agostinho, F.B.; Tubana, B.S.; Martins, M.S.; Datnoff, L.E. Effect of different silicon sources on yield and silicon uptake of rice grown under varying phosphorus rates. Plants 2017, 6, 35. [CrossRef] [PubMed]

49. Grieve, C.; Grattan, S. Mineral Nutrient Acquisition and Response by Plants Grown in Saline Environments. Agric. Ecosyst. Environ. 1999, 32, 203-229.

50. Del Amor, F.M.; Martinez, V.; Cerdá, A. Salt tolerance of tomato plants as affected by stage of plant development. HortScience 2001, 36, 1260-1263. [CrossRef]

51. Altuntas, O.; Dasgan, H.Y.; Akhoundnejad, Y. Silicon nutrition ameliorates salt stress of Capsicum annuum L. by ion regulation. In Proceedings of the XVIth EUCARPIA Capsicum and Eggplant Working Group Meeting in Memoriam Dr. Alain Palloix, Kecskemét, Hungary, 12-14 September 2016; pp. 465-469.

52. Chrysargyris, A.; Michailidi, E.; Tzortzakis, N. Physiological and biochemical responses of Lavandula angustifolia to salinity under mineral foliar application. Front. Plant Sci. 2018, 9, 489. [CrossRef]

53. Ali Turan, M.; Hassan Awad Elkarim, A.; Taban, N.; Taban, S. Effect of salt stress on growth and ion distribution and accumulation in shoot and root of maize plant. Afr. J. Agric. Res. 2010, 5, 584-588.

54. Adler, P.R.; Wilcox, G.E. Ammonium Increases the Net Rate of Sodium Influx and Partitioning to the Leaf of Muskmelon. J. Plant Nutr. 1995, 18, 1951-1962. [CrossRef]

55. Sharpley, A.N.; Meisinger, J.J.; Power, J.F.; Suarez, D.L. Root extraction of nutrients associated with long-term soil management Adv. Soil Sci. 1992, 19, 151-217.

56. Flores, P.; Carvajal, M.; Cerdá, A.; Martínez, V. Salinity and ammonium/nitrate interactions on tomato plant development, nutrition, and metabolites. J. Plant Nutr. 2001, 24, 1561-1573. [CrossRef]

57. Rahman, S.; Vance, G.F.; Munn, L.C. Salinity Induced Effects on the Nutrient Status of Soil, Corn Leaves and Kernels. Commun. Soil Sci. Plant Anal. 1993, 24, 2251-2269. [CrossRef]

58. Izzo, R.; Navari-Izzo, F.; Quartacci, M.F. Growth and mineral absorption in maize seedlings as affected by increasing nacl concentrations. J. Plant Nutr. 1991, 14, 687-699. [CrossRef]

59. Maas, E.V.; Ogata, G.; Garber, M.J. Influence of salinity on Fe, Mn, and Zn uptake by plants. Agron. J. 1972, 64, 793-795. [CrossRef]

60. Al-Harbi, A.R. Growth and Nutrient Composition of Tomato and Cucumber Seedlings as Affected by Sodium Chloride Salinity and Supplemental Calcium. J. Plant Nutr. 1995, 18, 1403-1416. [CrossRef]

61. Krauss, S.; Schnitzler, W.H.; Grassmann, J.; Woitke, M. The influence of different electrical conductivity values in a simplified recirculating soilless system on inner and outer fruit quality characteristics of tomato. J. Agric. Food Chem. 2006, 54, 441-448. [CrossRef]

62. Moya, C.; Oyanedel, E.; Verdugo, G.; Flores, M.F.; Urrestarazu, M.; Álvaro, J.E. Increased electrical conductivity in nutrient solution management enhances dietary and organoleptic qualities in soilless culture tomato. HortScience 2017, 52, 868-872. [CrossRef]

63. Zushi, K.; Matsuzoe, N. Metabolic profile of organoleptic and health-promoting qualities in two tomato cultivars subjected to salt stress and their interactions using correlation network analysis. Sci. Hortic. 2015, 184, 8-17. [CrossRef]

64. Ben, G.O.; Kafkafi, U. Melon fruit quality as affected by timing, duration, and concentration of phosphate and nitrogen sources in recycled hydroponic system. J. Plant Nutr. 2002, 25, 1563-1583. [CrossRef]

65. Petersen, K.K.; Willumsen, J.; Kaack, K. Composition and taste of tomatoes as affected by increased salinity and different salinity sources. J. Hortic. Sci. Biotechnol. 1998, 73, 205-215. [CrossRef] 
66. De Pascale, S.; Maggio, A.; Fogliano, V.; Ambrosino, P.; Ritieni, A. Irrigation with saline water improves carotenoids content and antioxidant activity of tomato. J. Hortic. Sci. Biotechnol. 2001, 76, 447-453. [CrossRef]

67. Serio, F.; De Gara, L.; Caretto, S.; Leo, L.; Santamaria, P. Influence of an increased NaCl concentration on yield and quality of cherry tomato grown in posidonia (Posidonia oceanica (L) Delile). J. Sci. Food Agric. 2004, 84, 1885-1890. [CrossRef]

68. Giuliano, G.; Bartley, G.E.; Scolnik, P.A. Regulation of carotenoids biosynthesis during tomato development. Plant Cell 1993, 5, 379-387.

69. Navarro, J.M.; Flores, P.; Garrido, C.; Martinez, V. Changes in the contents of antioxidant compounds in pepper fruits at different ripening stages, as affected by salinity. Food Chem. 2006, 96, 66-73. [CrossRef]

70. Toor, R.K.; Savage, G.P. Changes in major antioxidant components of tomatoes during post-harvest storage. Food Chem. 2006, 99, 724-727. [CrossRef]

71. Iglesias, M.J.; García-López, J.; Collados-Luján, J.F.; López-Ortiz, F.; Díaz, M.; Toresano, F.; Camacho, F. Differential response to environmental and nutritional factors of high-quality tomato varieties. Food Chem. 2015, 176, 278-287. [CrossRef]

72. Saito, T.; Matsukura, C.; Ban, Y.; Shoji, K.; Sugiyama, M.; Fukuda, N.; Nishimura, S. Salinity stress affects assimilate metabolism at the gene-expression level during fruit development and improves fruit quality in tomato (Solanum lycopersicum L.). J. Jpn. Soc. Hortic. Sci. 2008, 77, 61-68. [CrossRef]

73. Manzo, N.; Pizzolongo, F.; Meca, G.; Aiello, A.; Marchetti, N.; Romano, R. Comparative chemical compositions of fresh and stored vesuvian PDO "Pomodorino del Piennolo" tomato and the ciliegino variety. Molecules 2018, 23, 2871. [CrossRef]

74. Petric, T.; Kiferle, C.; Perata, P.; Gonzali, S. Optimizing shelf life conditions for anthocyanin-rich tomatoes. PLoS ONE 2018, 13, e0205650. [CrossRef] [PubMed] 